\title{
Das Big Data Game
}

\section{Zur spielerischen Konstitution kollaborativer Wissensproduktion in der Hochenergiephysik am CERN}

\author{
Anne Dippel
}

The Big Data Game. On the Ludic Constitution of the Collaborative Production of Knowledge in High-Energy Physics at CERN

This article looks at how games and play contribute to the big data-driven production of knowledge in HighEnergy Physics, with a particular focus on the Large Hadron Collider (LHC) at the European Organization for Nuclear Research (CERN), where the author has been conducting anthropological fieldwork since 2014. The ludic (playful) aspect of knowledge production is analyzed here in three different dimensions: the Symbolic, the Ontological, and the Epistemic. The first one points towards CERN as place where a cosmological game of probability is played with the help of Monte-Carlo simulations. The second one can be seen in the agonistic infrastructures of competing experimental collaborations. The third dimension unfolds in ludic platforms, such as online Challenges and citizen science games, which contribute to the development of machine learning algorithms, whose function is necessary in order to process the huge amount of data gathered from experimental events. Following Clifford Geertz, CERN itself is characterized as a site of deep play, a concept that contributes to understanding wider social and cultural orders through the analysis of ludic collective phenomena. The article also engages with Peter Galison's idea of the trading zone, proposing to comprehend it in the age of big data as a Playground. Thus the author hopes to contribute to a wider discussion in the historiographical and social study of science and technology, as well as in cultural anthropology, by recognizing the ludic in science as a central element of understanding collaborative knowledge production.

Keywords: Knowledge production, Collaboration, Competition, Play, Big data, Science and technology studies

Der vorliegende Artikel widmet sich der Frage, wie Spiele und spielen zur Big-Data-basierten Wissensproduktion in der Hochenergiephysik beitragen. Als Beispiel dienen Detektorkollaborationen am Large Hadron Collider (LHC) der Europäischen Organisation für Kernforschung (CERN), in denen die Autorin seit 2014 kulturanthropologische Feldforschung unternommen hat. Der ludische Aspekt der Wissensproduktion wird hier in drei verschiedenen Dimensionen analysiert: der symbolischen, der ontologischen und der epistemischen. Erstere verweist auf das CERN als Ort, an dem ein kosmologisches Wahrscheinlichkeitsspiel mithilfe von Monte-Carlo-Simulationen durchgeführt wird. Die Zweite wird durch kompetitive Infrastrukturen in der Arbeitspraxis verschiedener Kollaborationsgruppen sichtbar. Die dritte Dimension entfaltet sich durch ludische Plattformen, wie etwa Online Challenges und Citizen Science Games, die zur Entwicklung von machine learning algorithmen beitragen, deren Funktion notwendig ist, um die ungeheuren Mengen an Big Data verarbeiten zu können, die durch die Experimente gesammelt werden. Das CERN wird als Ort des Deep Play beschrieben, einem von Clifford Geertz entwickeltem Konzept, das dazu beiträgt, größere soziale und kulturelle Ordnungen durch die Analyse ludischer Kollektivphänomene zu begreifen. Der Artikel setzt sich darüber hinaus mit Peter Galisons Idee der Trading Zone auseinander und schlägt vor, diese in Zeiten von Big Data als Playground (Spielplatz) zu begreifen. Die Autorin hofft damit zu einer breiteren sozio-historiografischen Diskussion in den Science and Technology Studies (STS, Wissenschafts- und Technikforschung) und nicht zuletzt der Kulturanthropologie beizutragen, indem das Ludische in den Wissenschaften als zentrales Element für das Verständnis kollaborativer Wissensproduktion begriffen wird. 


\section{Wo das Spiel beginnt}

Zwischen Genfer See und dem Jura liegt die Europäische Organisation für Kernforschung (CERN), die derzeit größte Forschungseinrichtung für Teilchenphysik. Sie ist Schirmherrin eines Netzwerks von etwa 13.000 Menschen, die vor allem in den Arbeitsbereichen Physik, Technik, Ingenieursund Personalwesen tätig sind. Hinzu kommen Zulieferer, Sicherheits- und Servicekräfte, die auf dem CERN-Gelände ihre Arbeit verrichten. Es beherbergt mehr als 25 Experimente und Einrichtungen, an denen in kleinen, mittleren und großen Kollaborationen zwischen einigen wenigen bis $\mathrm{zu}$ mehreren tausend Menschen zusammenarbeiten. Im Jahr 1954 gegründet, ist die internationale Institution heute Hüterin und Herrscherin über das zweitgrößte Höhlensystem, das je von Menschen für wissenschaftliche Zwecke gebaut wurde. Hier befindet sich der Large Hadron Collider (LHC), ein Teilchenbeschleuniger, in dem Protonen gegenläufig rotieren und eine kinetische Energie von bis zu 6,5 Elektronenvolt erlangen, um bei nahezu Lichtgeschwindigkeit durch supraleitende Magneten gelenkt an vier spezifischen Stellen zu kollidieren, wo die Detektoren ATLAS (A Toroidal LHC Aparatus), CMS (Compact Muon Solenoid), ALICE (A Large Ion Collider Experiment) und $\mathrm{LHCb}$ (Large Hadron Collider beauty (LHCb) aufgebaut sind. Die Informationen über die Kollisionen werden in Geschwindigkeiten und Größenordnungen registriert, die für Menschen kaum vorstellbar sind. Mehr als 20 Millionen Protonenpakete kreuzen sich pro Sekunde, aber nur ein Bruchteil der Ereignisse wird durch die verschiedenen Subsysteme aufgezeichnet. ATLAS etwa filtert durch das Triggersystem aus einer Billionen Ereignissen während einer Kollision etwa einhundert heraus. ${ }^{1}$ Das Data Acquisition-System leitet Informationen zu den Speichern weiter, die durch das Grid, einem globalen Cloudsystem, auf hundert Computerzentren weltweit verteilt werden, wobei das größte davon auf dem CERN-Gelände selbst liegt. ${ }^{2}$ Das Dealen mit Big Data stellt somit eine der zentralen Aufgaben des LHCs dar.

Auch wenn physikalische Theorien innerhalb der einzelnen disziplinären Gebiete abgekoppelt von großen Modellen und der alles überschirmenden evolutionären Kosmologie der modernen Naturwissenschaften formuliert werden, erfahren die Besucher_innen etwa auf der ausgeschilderten Big Bang Tour, dass bei den Kollisionen nichts Geringeres als die Anfänge des Universums simuliert werden. Schließlich besitzen die von den Detektoren aufgezeichneten Kollisionen eine energetische Dichte, wie sie nach dem heutigen Stand physikalischer Theorie für die Zeit von etwa einem Billionstel nach dem Urknall angenommen werden - und lernen somit auf der Tour Legitimation von und Verbindungen zwischen einem der teuersten Experimente der Wissenschaftsgeschichte und dem gängigs- 
ten kosmologischen Modell für die Entstehung des Universums kennen. Es geht von der Explosion einer Singularität aus, in deren Nachfolge sich die Welt entfaltet hat, in der wir Menschen unsere Heimat haben. Bei der Betrachtung des Allerkleinsten geht es am LHC auf kosmologischer Ebene im anthropologischen Sinn um das große Ganze.

Während der ersten Laufzeit, zwischen 2009 und 2013 wurden experimentelle Messungen vorgenommen, die zum Nachweis des Higgs-Bosons geführt haben, einem zentralen Baustein des Standardmodells in der Physik. Seit dem Zusammenbruch der atomistischen Weltsicht, die von einem Unteilbaren kleinsten Baustein der Welt ausging, wurden mehr und mehr Teilchen im Inneren des Atomkerns entdeckt (die sich zugleich wie Wellen verhalten). Das Higgs-Boson selbst wird als quantenmechanischer Erregungszustand eines Feldes begriffen, das wiederum die Annahme des Higgs-Feldes bestätigt. Es erklärt, weshalb bestimmte Teilchen eine Masse haben, obwohl sie eigentlich keine haben dürften oder weshalb die schwache Wechselwirkung im Atomkern nur so eine kurze Reichweite besitzt. Seit 2015 und bis 2018 werden für die zweite Laufzeit des LHCs bei größeren Energien, besseren Aufzeichnungstechnologien und höherer Leuchtkraft sowohl Präzisierungen schon vorhandener Ergebnisse erlangt als auch neue physikalische Funde erhofft. Während im ersten Durchlauf ca. 100 Petabytes an Daten angefallen sind, wurden in der zweiten Laufzeit bereits im Juni 2017 aufgrund der Performance des LHCs und der erhöhten Luminosität über 200 Petabyte Daten gespeichert. ${ }^{3}$ Das CERN zählt damit zum größten Datenproduzent in der Wissenschaftswelt - und verfährt mit seinen ethisch unbedenklichen Daten, die nicht, wie etwa Datensätze von Google oder Facebook, Auskunft über Menschen geben, sehr offen. Computerspezialisten aus der ganzen Welt können das CERN Open Data Portal besuchen und mit den dort zur Verfügung gestellten Datensätzen ihre machine learning Algorithmen trainieren. ${ }^{4}$

Wie werden die "Cernians", oder "Cernies", scherzhaft nennen, mit diesen Massen fertig? Im Folgenden möchte ich anhand von Ergebnissen, die ich aus meiner laufenden ethnographischen Feldforschung seit 2014 gewinnen konnte, darlegen, dass das Big Data getriebene CERN auf verschiedenen Ebenen durch ludische Elemente gekennzeichnet ist. Zunächst werde ich seine symbolische Rolle über das Konzept des Deep Play beschreiben, an dem wie auf einer Bühne die Natur nachgespielt und sich dabei subatomaren Welten über Wahrscheinlichkeitsrechnungen angenähert wird. Im Anschluss werden die ontologischen Bedingungen wissenschaftlichen Arbeitens auf der Basis einer ludischen Infrastruktur beschrieben, in der agonale, das heißt, wettbewerbsorientierte Dynamiken der Kollaboration sowohl euagonale, als auch dysagonale Kräfte entfalten, also produktive, wie destruktive Aspekte von Kompetiti- 
on aufweisen. Erst aus dieser Synthese, des synagonalen Zusammenspiels verschiedener Disziplinen wird ein Playground (Spielplatz) geschaffen, der die Produktion von Wissen ermöglicht. ${ }^{6}$ Im letzten Teil des Aufsatzes soll der epistemische Einsatz von Spielformen, insbesondere ludischer Plattformen analysiert werden, bei denen der LHC, durch Expert Challenges und Citizen Science Games, sich eine größere Öffentlichkeit zu Nutze macht. Sie erlauben es, Machine Learning Algorithmen zu verbessern und produktiver mit der Masse von Big Data umzugehen. Der Aufsatz trägt somit insbesondere zur organisationstheoretischen Frage nach dem Wechselspiel von Kollaboration und Wettbewerb in wissenschaftlichen Communities bei, wie sie unter anderem in dieser Zeitschrift in einem Sonderheft jüngst diskutiert wurde (Nickelsen \& Krämer 2016; siehe auch Hackett 2005; Hagstrom 1965; Shrum 2005; Steingart 2012) und erklärt dabei, wie wissenschaftliche Innovation entsteht (Nowotny 2006, 2008), dass sich der wechselseitige Aushandlungsprozess in den Wissenschaften (Felt et al. 1995) immer wieder lokal ausgestaltet und die Balance von Vertrauen und Skepsis situativ ausgehandelt wird.

Die Beispiele zeigen, wie sich die von Peter Galison beschriebene Trading Zone - ein Zwischenbereich in dem Wissensprozeduren lokal koordiniert werden können, selbst wenn größere Bedeutungsvorstellungen dabei aus gegensätzlichen Richtungen aufeinandertreffen (Galison 1997: 46) - in Zeiten von Big Data am CERN in einen Playground verwandelt. Ähnlich den Aushandlungsprozessen von sozialen Hierarchien und lokal definierten Regeln, Habitus und Codes auf Spielplätzen, arbeiten Physiker_innen, Ingenieur_innen und Computerwissenschaftler_innen zusammen, teilen sich „Spielzeuge“, Tools und Toys, und kommunizieren mithilfe eines sich von Werkzeugen her entfaltenden Vokabulars, auf der Basis geteilter globaler Sinn- und Bedeutungswelten. So entstehen die von Susan Leigh-Star und John Griesemer beschriebenen Boundary Objects (Star \& Griesemer 1989), durch das, was Sheila Jasanoff als Co-Production (2004) bezeichnet. Die Architektur und Infrastruktur algorithmischer Wissenskulturen unterscheidet sich von der klassischen Trading Zone durch Möglichkeiten, die sich etwa mit Hilfe des World Wide Web, leistungsstarken Servern und Rechnern, gigantischen Datenfarmen und der Ubiquität des personalisierten Computers bieten. Die Infrastruktur bildet, mit Janet Vertesi gedacht, Seamful Spaces, da hier Menschen, Maschinen und Daten in multiplen, koexistierenden Umwelten miteinander „versäumt“ werden und durch diese infrastrukturellen „Verpflichtungen“ kollaborieren (Vertesi 2014). Die unglückliche Dichotomie von virtuell und analog wird in solchen digitalen Arbeitsumwelten somit hinfällig, das Zusammenspiel von Menschen, Maschinen, Algorithmen und Daten beschreibbar. Während in der Trading Zone überschaubare Komplexität ausgehandelt wird, ist in Seamful Spaces 
die Komplexität der Kollaboration derart verdichtet, dass Spielförmigkeit überhaupt Handlungsfreiheit erlaubt. Dabei entsteht weniger ein Kreol, als dass fachspezifische spontane Übersetzungen vorgenommen werden. "CERNglish" wandelt sich von Generation zu Generation, von Playground zu Playground und erhält im Gegensatz zu Kreolsprachen seine Stabilität aus materiellen Bedingungen und der physikalischen und informationstheoretischen Terminologie.

Auf den Playgrounds der Hochenergiephysik wird Wissen ausgehandelt, das hohen Anspruch besitzt. Werbebroschüren, pädagogischen und populärwissenschaftlichen Vorträgen folgend, geht es um etwas Faustisches, nämlich, zu ergründen „was die Welt im Innersten zusammenhält“. Für die Physik haben solche Worte, entrissen der Schlüsselszene des Goethe'schen Theaterstücks, in der Faust seine Seele an den Teufel verkauft, um Erkenntnis zu erlangen, keine größere Bedeutung. Aus kulturanthropologischer Sicht jedoch lässt sich hier, neben üblichen Marketingstrategien, viel über das Selbstverständnis der Physik ablesen. Sätze wie diese geben den imperialen Anspruch der Physik preis, über soziale und kulturelle Welt- und Wertvorstellungen einer Gesellschaft eine hegemoniale Deutungsmacht zu beanspruchen. Während nach der Beschaffenheit einfachster Strukturen gesucht wird, geht es doch um nicht weniger als Wahrheit mit einem Konzept von Natur zu beanspruchen, die sich messen, mathematisch beschreiben und reproduzieren lässt.

\section{Die Regeln des Playgrounds}

Seit Jahrzehnten wird die Hochenergiephysik von Wissenschaftshistoriker_innen, Kulturanthropolog_innen und Forscher_innen aus dem Bereich der Science and Technology Studies befragt. Während die historischen Bedingungen aus organisationsgeschichtlicher Perspektive (Hermann et al. 1987, 1990; Krige et al. 1996), im Spannungsfeld von internationaler Wissenschaftspolitik (Krige 2006) und nationalen Interessen (Carson 2010; Strasser 2009) sowie im Blick auf wissenschaftsgeschichtliche Entwicklungen (Galison 1997) untersucht wurden, widmeten sich soziologische Studien insbesondere dem Netzwerk von menschlichen und technischen Akteuren (Knorr-Cetina 1984, 1999) oder der Organisation des Wissens (Graßhoff \& Wüthrich 2012). Darüber hinaus wurde auch das Miteinander verschiedener Forscher_innen unterschiedlicher kultureller Herkunft am Beispiel nationaler Stile erforscht (Nothnagel 2001), der Habitus und die Lebenswelt der Hochenergiephysik beschrieben (Traweek 1988) und die wissenschaftlichen Werkzeuge ebenso wie ihre epistemischen Möglichkei- 
ten und anthropozentrischen Beschränkungen (Roy 2012, 2014) aus philosophischer und wissenssoziologischer Sicht befragt (Borelli 2014; Merz 2002).

Die bisherigen Forschungen zur Wissensproduktion und Arbeitsorganisation am CERN haben sich der Betrachtung ludischer Elemente nur peripher gewidmet. Max Boisot und Michael Nordberg zum Beispiel verweisen auf die Implementierung von spieltheoretisch basierten Nash-Equilibria $^{7}$ für kollektive Managementstrategien in der ATLAS-Kollaboration, wenn es darum geht, Richtlinien auf der Basis von Spieltheorie zu entwickeln. So verweisen die Autoren darauf, dass die ATLAS-Kollaboration flache Hierarchien etabliere und einen demokratischen Prozess, der rationale Entscheidungen für Konflikt- und kooperative Situationen favorisiere. Sie führen aus, wie für ein reibungsloses Funktionieren am CERN Managementstrukturen etabliert wurden, die Mitarbeiter_innen als „Player“ begreifen, deren Strategien individualisiert und verdeckt werden (Boisot \& Nordberg 2011: 104). Der wirtschaftswissenschaftlich ausgerichtete Blick verdeckt jedoch die Frage nach Dynamiken, die sich mit dem ökonomischen Denkstil bloß unzureichend erklären lassen. Hier rückt die regulierende und kontrollierende Funktion von Spielen, die funktionale Relation von Kosten und Nutzen, in das Zentrum der Aufmerksamkeit und verdeckt den Überschuss an Energie und Imagination, der das CERN zu dem magischen „Mekka der Physik“ macht, als dass es von vielen subjektiv beschrieben und wahrgenommen wird. Diese Elemente sind indessen typisch für das, was ich als Playground bezeichne. Auch die Wissenssoziologin Karin Knorr-Cetina greift die kontrollierende Seite des Spiels auf und verweist auf die Bedeutung von Kriegs- und Planspielen als Vorlage für Laborforschungen (1999: 32-35, 111-135). In ihrer Studie zur Arbeit theoretischer Physiker_innen am CERN verzeichnen Martina Merz und Karin Knorr-Cetina Interviewpassagen, in denen Informant_innen auf die Kreativität und imaginative Kraft des Ludischen verweisen, indem sie ihre alltägliche Arbeit an den Gleichungen als „herumspielen“ bezeichnen und ihre Disziplin als einen „unendlichen Spielplatz“ der Mathematik (Merz \& Knorr-Cetina 1994: 13) beschreiben. Die Autorinnen verfolgen dieses Phänomen jedoch nicht weiter, sondern widmen sich in ihrem Aufsatz den funktionalen Formen theoretischer Wissensprozesse.

Die Größe einer Gemeinschaft besitzt zentrale Bedeutung für den Gegenstand der Erkenntnis, wie unlängst die Anthropologin Nurit Bird-Davis dargelegt hat (2017) - ebenso wie der Einsatz von „sociotechnical imaginaries" (Jasanoff \& Kim 2013), die es überhaupt erst ermöglichen, dass Kosmologien (Sahlins 1996) entstehen können. Das CERN kann mit seinen etwa 13.000 Mitgliedern zwar als eine medium-scale Community begriffen werden. Ihre Konzepte und ihr Habitus aber werden dadurch, dass 
viele Cernies später gar nicht in der Wissenschaft arbeiten, sondern in anderen globalen Communities, vor allem in die Welt der datenanalytischen Marktwesens getragen. Und doch bildet die Hochenergiephysik einen Mikrokosmos in dem kulturelle und soziale Phänomene zu einem kosmopolitischen Ideal amalgamiert sind, an dem seit seinem Bestehen die imaginativen Kräfte nationaler Identitäten ebenso wie soziale Herkunft von der Identität des Ortes ausgeglichen werden, selbst wenn diese im Alltag und für jedes Individuum weiterhin eine besondere Rolle spielen und oft durch kulturelle Gruppenbildungen ausgelebt werden. Sharon Traweek hat argumentiert, dass insbesondere die Hochenergiephysik eine Trennung von Wissenschaft und Gesellschaft vorantreibe, indem sie eine „Kultur der Nichtkultur" propagiere, die danach strebe, eine Welt ohne Temperament, Gender, Nationalismus und Unordnung, außerhalb von menschlicher Raum und Zeit zu fördern (1988: 162). Im Gegensatz dazu scheint mir jedoch, dass von Physiker_innen im Allgemeinen und Cernies im Besonderen von einer transnationalen und kosmopolitischen Kultur gesprochen werden kann (Pheng \& Robbins 1998; Beck 2005), deren Alltagssprache sich zwar - wie jede orale Sprache - rasant wandelt, aber deren seit Generationen weitergegebene soziale Codes und epistemische Wissensbestände einen spezifischen Wertekanon und eigenen kulturellen Habitus herausgebildet hat. Dabei unterscheiden sich die gelebten Codes von gesetzten Normen und wissenschaftlichem Verhalten im Alltag. Eine kulturanthropologische Analyse dieser Kultur erscheint daher ebenso fruchtbar, wie die Auseinandersetzung mit „Kulturklassikern“ wie zum Beispiel etwa den Arawete, den Berbern, den Deutschen, Griechen, Nuern oder Trobriandern. Für das digitalisierte Zeitalter liefert insbesondere die Kultur der Physik Erkenntnisse, schließlich wird hier seit den 1970er-Jahren die Automatisierung von Arbeit vorangetrieben (Galison 1997) und an wenig anderen Orten hat sich der Personal Computer derart früh als ubiquitärer Arbeitsgegenstand etabliert wie hier.

Während meiner Arbeit unter Physiker_innen, die mir die Möglichkeit bietet sowohl in virtuellen Gruppen als auch im realen Alltag inmitten der technischen Welt und an den Arbeitsplätzen der Cernies „herumzuhängen“ (Schwanhäußer 2015), konnte ich aus der teilnehmenden Beobachtung alltäglicher Praxis (Knecht 2013; Niewöhner et al. 2012) die vorherrschenden Annahmen in der Wissenschaftsforschung über die Arbeitsweise in der Hochenergiephysik um den Aspekt des Ludischen erweitern. Die bisherige Forschung betrachtet wissenschaftliches Arbeiten überwiegend von den Konzepten der epistemischen Kulturen, der Akteur-Netzwerk-Theorie, der Co-Production und dem Konzept der Trading Zone, in der interdisziplinäres Wissen ausgehandelt wird und sich reziprok konstituiert. In den vergangenen Jahren ist insbesondere das Konzept der Akteur-Netzwerk- 
Theorie aufgrund seiner liberalen Logik in Kritik geraten, die funktionalistische Aspekte und Nutzenrechnungen in den Vordergrund stellt sowie die Agency von Menschen in einem größeren soziotechnischen Apparat erscheinen lässt, ohne Machtverhältnisse in den Blick zu nehmen. Desgleichen entbehrt auch das Konzept der Trading Zone nicht a priori gesetzten Grundannahmen gegenwärtiger liberaler Ökonomien, wird doch der Handel essentialisiert und mit der sprachlichen Kreolisierung verknüpft, einem Konzept, das seinen Ursprung in von Kolonialismus geprägten Machtverhältnissen verortet. Das „Laboratory Life“ (Latour \& Woolgar 1986) stellt mehr als bloßes Netzwerk von nichtmenschlichen und menschlichen Akteuren einer Wissensfabrik dar: Wo Menschen am Werk sind und Vorstellungskraft gefragt ist, kommt das Spiel zum Tragen. Unvorhersehbares wird gefunden, indem das Vorhergesehene widerlegt wird. Erfolgreich zu sein im „Spiel der Wissenschaft“ (Popper 1935: 32), wie Popper seine Logik auch umschrieb, heißt somit, individuell $\mathrm{zu}$ agieren und sich doch kollektiven, spezifischen Sprachen, Ritualen, Regeln sowie auch Habitus zu unterwerfen. Spielen ist einer der effizientesten Wege schnell, effektiv und dabei überzeugend Ergebnisse nach Zeitplan zu liefern, denn Spiel verstärkt die sowieso schon durch gemeinsame Praxis, Ideen und Zeitregimes produzierten Bindekräfte und den dadurch ausgelösten Vergemeinschaftungsprozess. Die wissenschaftliche Wirklichkeit als Spiel zu begreifen, hilft innerlich Abstand zu nehmen, schafft den Raum für Imagination und Falsifikation ebenso wie sie den gesellschaftlichen Druck mildert, der auf Mitgliedern einer Arbeitskollaboration wie dem CERN lastet, das pro Jahr in etwa eine Milliarde Euro an öffentlichen Geldern kostet.

Arbeit im Labor und Wissenschaft zu machen ist beides: Ein kreativer Prozess der Wahrheitsfindung und eine Organisationskultur. Experimente und wissenschaftliches Arbeiten sind, ebenso wie Spiele, von Victor Turner als „liminoide“ Situation beschrieben worden, also freiwillig gewählte Schwellensituationen (Turner 2009). Wissenschaften erhalten, wie Johan Huizinga in seiner Studie Homo Ludens bemerkte, durch ihre Abgeschlossenheit und Regelhaftigkeit Spielcharakter (Huizinga [1938] 1992). Sie schaffen Realität in Realität. Wurde in der Wissenschaftsforschung außer beispielsweise in den Arbeiten von Paula Findlen (1998) sowie einigen Randüberlegungen von Mara Beller (1998) und Paul Forman (1989) zur Bedeutung von Ironie, dem Ludischen bloß geringe Aufmerksamkeit beigemessen, so hat sich auch die anthropologische Forschung zum Spiel in den vergangenen dreißig Jahren nur schleppend entwickelt. Arbeiten und Spielen werden im Alltag häufig als oppositionelle Tätigkeiten definiert, die den Menschen existentiell und ontologisch bestimmen: Während durch Arbeit die Welt zum Gegenstand gemacht und das Selbst überhaupt im Zusammenhang mit Anderen durch Arbeit entsteht (grundlegend hierzu: 
Marcuse 1933), befreit sich der Mensch mit dem Spiel von den Zwängen der Notwendigkeit (Bally 1966) und enthebt sich der Bedingungen des Existentiellen. Wurde das Spiel somit lange Zeit als Gegensatz zur Arbeit oder zum Kampf begriffen, konnte in der Kulturanthropologie unter anderem durch Philipp Stevens das Verhältnis als falsche Dichotomie (Stevens 1980) beschrieben werden, da das erste ein „Modus menschlicher Erfahrung“, während Letzteres eine „Form der Aktivität“ (Malaby 2009: 208) darstellt. Zugespitzt gefasst: Spiel ist eine ontologische, Arbeit eine existentielle Praxis. Auch Casey O’Donnells (2014) Ethnographie über die wechselseitige Verschränkung von Arbeit und Spiel in kollaborativen Arbeitspraxen von AAA-Videospiel-Entwicklern sowie T.L. Taylors Beobachtungen von „Arbeit/Spiel-Überlagerung“ im Kontext der Professionalisierung von digitalem Spiel auf der E-sport Bühne (Taylor 2012) verfolgen eine ähnliche Stoßrichtung, ebenso die anthropologischen Überlegungen von David Graeber (2013, 2015). Die vorliegende Forschung stellt somit ein Desiderat dar, das in Zeiten der Gamification und der rasanten Etablierung einer neuen Disziplin mit Namen "Game Studies“ nach dringlicher Bearbeitung fragt.

\section{Deep Play @ CERN: Symbolische Variation}

Das CERN ist unbenommen ein Ort der Superlative, der Forscher_innen, Student_innen, Journalist_innen und Tourist_innen aus aller Welt anzieht. Allein 30.000 Schüler_innen besuchen pro Jahr das CERN. Die meisten begegnen zunächst einem kugelförmigen, ikonischen Gebäude, dem Globe of Science and Innovation, blicken zu den im Wind aufgereihten Mitgliedsflaggen hoch, hinter denen ein unscheinbar wirkender Fußgängereingang liegt, der die Tore zu einem Labyrinth von Laboren, Büros und Unterkünften freigibt, in funktionellen, grauen Gebäuden und Hallen aus den letzten fünfzig Jahren untergebracht. Der streunende Blick einer Anthropologin jedoch versucht hinter die Fassaden zu blicken und bleibt zu Beginn an bekannten Klischees hängen, so etwa der Shiva-Skulptur, die unter Verschwörungstheoretiker_innen berüchtigt ist (Abb. 1). Sie steht auf dem Square Eldorado, eingepasst zwischen dem Housing Service und Gebäude 40, das die ATLAS und CMS Geschäftsstellen beherbergt, ${ }^{8}$ die zwei „VorzeigeKollaborationen" und Geschwisterexperimente des LHC. Am Tage thront sie zwischen grünen Weinbergen auf der einen und der CERN Cafeteria auf der anderen Seite. In der Nacht vollführt ihr von Scheinwerfern vergrößerter Schatten an der Schwelle zwischen Licht und Dunkelheit auf den gegenüberliegenden Wänden ein theatralisches Spiel, während die Strom- 


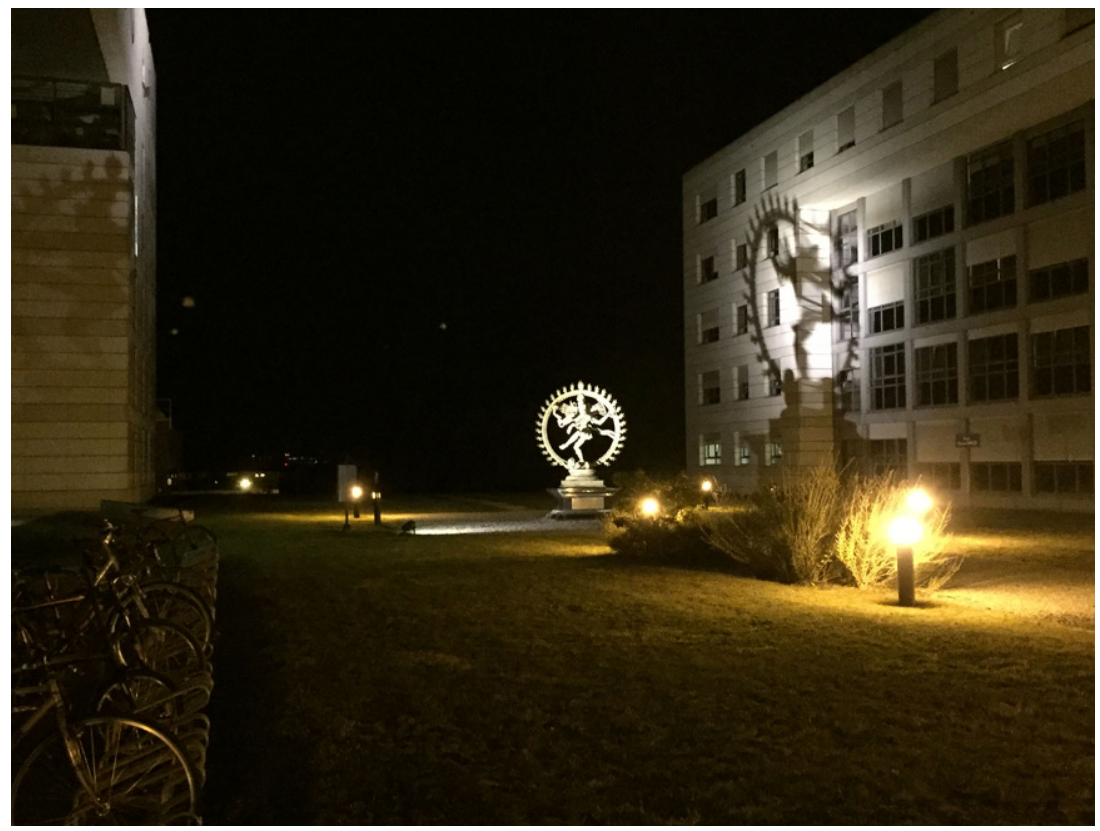

Abb. 1 Shivas Schatten am Gebäude 42 (Foto A. Dippel)

leitungen surren. Der Schöpfer und Zerstörer wird in der Nataraya-Pose dargestellt. Shiva tanzt den Tandava. Der Hinduphilosophie folgend, verkörpert er die Quelle für den Zyklus von Werden, Erhalten und Vergehen. Shiva, von dem ein anderer Mythos erzählt, dass er mit seiner Frau Parvati unendlich lang ein Würfelspiel spiele, ist die einzige Manifestierung einer Weltreligion die es ans CERN geschafft hat.

Aber warum überhaupt steht die Skulptur dort, schwer zu übersehen und scheinbar irrelevant? Ursprünglich war sie ein Geschenk des indischen Ministeriums für Atomenergie, das 2001 übergeben und feierlich aufgestellt wurde. Die meisten Cernies schenken der Statue keine große Aufmerksamkeit, denn Religion spielt in ihrem Arbeitsalltag kaum eine Rolle. Ihnen geht es um Teilchenphysik, um Begriffe wie Machine Learning, Luminosität oder Pile-up von Daten. Für manche ist die Statue daher bloß „eine Möglichkeit das tote Ende einer Einbahnstraße zu verdecken, weil es für die Wissenschaft hier nicht weitergeht“, oder „ein Zeichen, dass CERN kein Geschenk zurückweisen kann“. „Es passt gut in das Bild“, sagen andere und, „es ist eine gute Metapher für das, was wir Physiker hier jeden Tag tun." Insbesondere in den letzten beiden Kommentierungen lassen sich interessante Verbindung zwischen Shiva, dem kosmischen Tänzer und einer Kollaboration tausender Cernies herstellen, die in ihren Theorien und Experimenten die Anfänge des Universums inszenieren. Denn sie tun das 
vor allem, indem sie rechnen und kodieren und ganz so wie Shiva, dem die Materie zum Spielen zur Verfügung steht, verrichten Physiker_innen diese Arbeit auf der Basis eines Wirbeltanzes von Teilchen, durch MonteCarlo Simulationen von Big Data. Im Grunde „spielen“ Physiker_innen, so wie Shiva und Parvati, ein imaginäres Würfelspiel, wenn sie „fundamentale“ Eigenschaften von Natur mit „Monte-Carlo-Methoden“, „Pseudo-Zufallszahlen" und Toy Simulations vorhersagen. Ihre Datenmassen sammeln sie durch die gezielte Zerstörung von Protonenpaketen, die dabei Spuren in Siliziumlagen und Teilchenkammern sensibler Detektoren hinterlassen, welche „einem Mandala gleichen. Wunderschön, aber im Grunde weiß niemand wirklich was sie bedeuten oder was damit anzufangen ist", wie Michael, ein älterer Hochenergiephysiker, meint.

Physiker_innen am CERN erzeugen, erhalten und zerstören so wie Shiva Materie und Antimaterie, modellieren und simulieren Ursprung und Essenz des Kosmos, mit anderen Worten, sie machen Physik. In seinen eigenen Werbetexten existiert das CERN, um das „Mysterium der Natur“ zum „Nutzen der Menschheit" zu „verstehen“.9 Die Forschungsstätte wurde deshalb auch als ein „Projektionsort“ bezeichnet (Houdart 2013): Das CERN bietet durch die hier zu beobachtende Verdichtung unterschiedlichster Kulturen und gemeinsam geteilter Wertvorstellungen, die alle an der Arbeit an einem Weltbild mitwirken, einen Ort auf den sich Hoffnungen, Ängste von Menschen projizieren und sich die moderne Welt reflektieren lässt. Kann das CERN nicht auch als ein Ort des Ludischen begriffen werden, an dem ein kulturelles Deep Play (Geertz 1973) exerziert wird, weil sich Wünsche und Träume einer Gesellschaft nach einer friedlichen transnationalen Welt - gleichgültig durch welche internationalen politischen Entwicklungen hervorgerufen (Krige 2006, 2016) - dadurch verwirklichen, dass gemeinschaftlich an ungeheuerlichem Wissen geforscht wird und tagtäglich mühsam kulturelle Gräben auf der Basis des Spiels der Wissenschaft (Bourdieu 1987: 122) überwunden werden?

Clifford Geertz' Konzept des Deep Play beschreibt den traditionellen balinesischen Hahnenkampf, bei dem Hähne in einer als ausnehmend friedlich bekannten Gesellschaft stellvertretend für die Männer selbst kämpfen, als „metasocial commentary upon the whole matter of assorting human beings into fixed hierarchical ranks and then organizing the major part of collective existence around that assortment" (1973: 448). Ganz ähnlich wie der balinesische Hahnenkampf liefert der Ringbeschleuniger des CERN einen metasozialen Kommentar über das friedfertige, kulturspezifische Unterfangen in liberalen Zeiten, nicht-menschliche Entitäten nach fixen, stratifizierten und symmetrisierten Systemen anzuordnen, um dadurch die kollektiven Vorstellungen über den Kosmos zu organisieren. In seiner Ethnographie Negara erweitert Geertz seine Spielrhetorik auf das 
gesamte politische System Balis. Er beschreibt es als eine Form des sozialen und kulturellen Mimikrys:

[S]tate ceremonials of classical Bali were metaphysical theatre: theatre designed to express a view of the ultimate nature of reality and, at the same time, to shape the existing conditions of life to be consonant with that reality; that is, theatre to present an ontology and, by presenting it, to make it happen - make it actual (Geertz 1980: 104).

Um das Gemeinschaftswesen der Balinesen zu verstehen, konzentriert sich Geertz auf Spiele und Rituale. Jedes soziale Mimikry wird hier zur kulturellen Mimesis, ein Begriff, der nach Plato einen „Akt der Komposition“ bezeichnet, der in einem „Akt der Schöpfung mündet, und von einem Schauspieler, der Sprachrohr oder Rezitator ist, vorgeführt wird“ (Havelock 1963: 22, Übs. d. A.). So wie beim Hahnenkampf in Bali gesellschaftliche Rollen und Werte ausgehandelt, bzw. vorgeführt werden - ist das CERN eine transnationale Arena, in der sehr viel mehr „auf dem Spiel“ steht, als „Physik“. Es geht um einen kosmopolitischen Traum, um Ideen wie „Europa“, „Völkerfrieden“ und „Grundlagenforschung“. Zugleich ist das CERN eine idealisierte Gemeinschaft. Hier werden managementbasierte Strukturen und ökonomische Theorien soziale Wirklichkeit. Im Umgang mit Big Data, der Konstitution von Open Platforms und der Arbeit mit neuronalen Netzen spiegeln sich soziale Hierarchien und die Wertigkeit von Wissen in einer posthumanistischen Kultur, die Technik und Naturwissenschaft über den klassischen Bildungskanon stellt. Oder der gerne im CERN kolportierte Mythos, demzufolge Papst Franziskus bei einem Treffen zum damaligen Generaldirektor des CERN Rolf-Dieter Heuer gesagt haben soll: „Herr Heuer, ich weiß gar nicht, wo das Problem liegt: Sie beschäftigen sich mit allem, was nach dem Big Bang passiert ist, wir mit allem, was davor kommt." Das Naturverständnis der Experimentalphysik produziert eine Gemeinschaft von Akteuren, die Werte, Handlungs- und Denkweisen vereint. Habitus und Arbeitsstil werden von den Kulturtechniken hier erst hervorgebracht und durch das disziplinäre Selbstverständnis (Traweek 1988) verstärkt.

Wir haben gerade gemeinsam im Restaurant 1 ein dreigängiges Mittagsmenü gegessen, Salat und Joghurt inklusive. Es ist inzwischen Zeit zurück an den Arbeitsplatz im Building 40 zu gehen, wo wir kurz vor der Rückkehr noch einen Espresso holen wollen. Achtlos laufen wir an der Shiva-Skulptur vorbei. Sebastian, der inzwischen seit über zehn Jahren am CERN arbeitet, dessen Arbeit an Algorithmen in der ganzen ATLAS-Kollaboration bekannt ist, erklärt euphorisch: „Mit Simulationen haben wir endlich die Möglichkeit, wirklich etwas zu schöpfen. Für ihn ist seine Arbeit „Gott spielen mit Hilfe des Computers“. Wieder einmal verrät das, was 
so daher gesagt ist, doch etwas über das Selbstverständnis und lässt den „Gottkomplex“, der sich mit der technischen Beherrschung von Welt in das naturwissenschaftliche Denken und Ingenieurswesen eingeschrieben hat, durchscheinen. Der Zusammenfall von „Gott“ und „Spielen“ steht hier zur Diskussion, denn nur den Göttern ist es erlaubt, ohne Rücksicht auf Verluste zu spielen, egal ob sie gewinnen oder verlieren, sie sind unsterblich (Graeber 2015: 296). Regel, Anfang und Ende, sowie alles was dazwischen zählt, ist Menschenspiel - nur in diesem Rahmen verlieren Handlungen ihre existentielle Bedeutung. Cernies spielen wie Götter, schaffen, erhalten, modellieren und zerstören Teilchen, wobei sie Erkenntnisspiele vollführen, um Muster zu entdecken. „Simulationen des Detektors etwa, dienen dazu, die Messung zu optimieren. Sie ist ein Mittel, um die Natur nachspielen zu können. Durch Simulation wissen wir mehr über die Natur," sagt Christian, ein erfahrener Post-Doc, der an der Simulation vom ATLAS-Detektor arbeitet, „es ist ein Parallelsystem von Natur."

Die am CERN ausgeführten Experimente wurden bisher als Deep Play beschrieben, weil sie einen symbolischen Schauplatz für die naturwissenschaftliche Kosmologie darstellen, indem sie eine Miniatur physikalischer Kosmogonie repräsentieren und von Steuergeldern finanziert dem öffentlichen Interesse ausgesetzt sind. Da in der Wissenspraxis Physiker_innen gleich Göttern mit einer Welt in einem liminoiden Zustand spielen, in dem es um alles geht, aber nichts existentiell zählt. Zweiundzwanzig Europäische Mitgliedsstaaten sowie Universitäten und Nationen aus der ganzen Welt unterstützen die Organisation. Der Ort ist ein gelebtes Beispiel der kosmopolitischen, globalisierten, internationalisierten und liberalen Gesellschaft mit ihrer kulturellen Ausrichtung auf Mathematik, Informatik, Naturwissenschaft und Technik (MINT) als Antriebskräfte gesellschaftlicher Entwicklung. Auf der Basis dieser Quadrarchie zielt das CERN darauf, mit Hilfe seiner technologischen und organisationalen Infrastruktur, überprüfbare Modelle zur Natur der Natur zu liefern.

\section{Ludische Infrastrukturen: Ontologische Variation}

Der Physiker Harald Lesch sagte in einer Fernsehshow über den Erfolg der Organisation am Fuße des Jura: „Wieso das CERN so gut funktioniert? Einfach weil dort alle spielen“. ${ }^{10}$ Das Wort „spielen“ wird von den Physiker_innen alltäglich und selbstverständlich benutzt. Es ist ins Unbewusste des Vokabulars der hochenergiephysikalischen Alltagssprache eingebettet und wirkt wie ein Kontrastmittel zum technischen, computerwissenschaftlichen und in seinen Ursprüngen vielfach ballistischen Vokabular, das den 
Alltag beherrscht. Während „Spielen“ am CERN auf der ontologischen Ebene wie oben dargelegt wurde dank der imaginären Möglichkeiten der Simulation in einen gottähnlichen Modus versetzt, erscheint es auf der alltäglichen Ebene als ontologischer „Erfahrungsmodus“ (Malaby 2009: 208) insbesondere als Ort des Agon, des Wettkampfes. Götter spielen um des Schicksals willen, auf Verluste müssen sie keine Rücksicht nehmen. Gesellschaften investieren in Experimente um Wahrheit zu finden, und wie es so schön heißt, hat jede Wahrheit ihren Preis, der in diesem Fall durch Steuergelder bezahlt wird.

Ludische Arbeitspraxen können in vielen Bereichen der Kollaboration beobachtet und entdeckt werden, gerade weil die Physiker_innen ihr Tun mit „heiligem Ernst“ im Sinne Johan Huizingas betreiben (1992 [1938]: 20-23, 186). Insbesondere die Fähigkeit enorme Massen an Big Data zu verwalten und eine Vielzahl von Menschen und Maschinen miteinander $\mathrm{zu}$ verknüpfen, lässt die Trading Zone zum Playground werden. Auf dem beispielsweise von Karin Knorr-Cetina diagnostizierten Wissenschaftsmarkt, in dem Wissenschaftler_innen als „economic reasoner“ erscheinen (KnorrCetina 1984: 74), müssen sie heute als „ludic reasoner" auftreten, die Herausforderungen suchen, Risiken eingehen und deren individuelles Gewinnstreben eben nicht von materiellem Profit getrieben ist (aber materiellen Zwängen unterworfen ist), sondern davon zu erkennen, das Spiel des Wissens zu spielen und die eigene Sache so gut wie möglich zu machen.

Wettbewerb erscheint heutzutage für Wissenschaftskulturen im Besonderen wie für die Gesellschaften einer liberalen, globalisierten Marktwirtschaft im Allgemeinen emblematisch und wird insbesondere aus wirtschaftswissenschaftlicher Perspektive analysiert. Aus kulturanthropologischer Sicht kann Wettbewerb in vielen Sphären beobachtet werden und muss von den Ergebnissen der Spielforschung her besehen zunächst als eine Spielform begriffen werden. Die „agonistische Struktur“ (Caillois 2001: 9-13), lässt sich nicht bloß auf der Mikroebene als „Konkurrenz“ zwischen rivalisierenden Wissenschaftler_innen beobachten, sondern ist konstitutioneller Bestandteil der Kollaborationen selbst. Am Deutlichsten lässt sich das anhand der Zwillingskonstitution von ATLAS und CMS am LHC veranschaulichen, deren Produktivität als Teil einer Wissensmanufaktur beschrieben wurde (Knorr-Cetina 2002), deren ludischer Elan und die in der Kollaboration akkumulierte individueller Lust und Hingabe ans Forschen nach genauerer Betrachtung fragt.

Auch wenn ein ökonomischer Grund für die Implementierung des Doppelexperiments ausschlaggebend war, da die Experimente so teuer und energieaufwändig sind, dass ein paralleler Aufbau von Experimentalkollaborationen viel kostengünstiger und effizienter wird, trägt diese Arbeitsarchitektur zur Wettbewerbssituation bei und soll bewirken, dass die Ex- 
perimente schneller zu verwertbaren und präsentierbaren Forschungsergebnissen gelangen. Jedes Experiment vermag die Ergebnisse des anderen unmittelbar zu überprüfen und $\mathrm{zu}$ falsifizieren. Erst wenn beide das Gleiche bestätigen, hat die Messung Anspruch auf Faktizität. Und schon deshalb bekommt die Frage, wer von beiden Kollaborationen warum als erstes etwas aus den Datenmassen gefischt hat, einen Stellenwert zugeschrieben. Der Wettkampf um die Beantwortung hunderter spezifischer wissenschaftlicher Fragestellungen im Blick auf Energie, Masse, Form und Zerfallszeit von kollidierenden Teilchen versetzt die Menschen in einen immersiven Rausch, der gerade durch das gemeinsame Zusammenarbeiten im Wettlauf mit einer anderen Gruppe verstärkt wird. Nur im Scherz nennen sich Physiker_innen aus rivalisierenden Kollaborationen „Feinde“: In der Realität floriert der Austausch zwischen den Gruppen und sie nehmen den Wettkampf „sportlich“, wie Valentina sagt, die eine hohe Position bei ATLAS bekleidet. So bleibt die Arbeit „spielerischer Ernst“ und wird nie zu „bitterem Ernst“, der die produktive Dynamik der Zwillingsstruktur zerstören würde.

Physiker_innen möchten „innerhalb der strengen Beschränkungen von Formeln und technischen Randbedingungen Wissen. Spielen ist für uns die Grundlage für Vorstellungskraft", sagt die Doktorandin Marlene auf einer Party zu mir, während sie einen Flaschenöffner in der Hand hält - eine kleine Muskelmannfigur, bei der anstelle eines primären Geschlechtsmerkmals einen Korkenzieher sitzt, den man aus- und einklappen kann - „Habe ich mal von einer Kollegin geschenkt bekommen. Ist total lustig, oder?“ Sie lacht. „Ein nützlicher Mann.“ Dann führt sie weiter aus: „Wir müssen zum Beispiel sehen, dass die Algorithmen funktionieren, aber welche Physik die für ihre Berechnungen verwenden, wissen wir nicht. Die sind nicht-interpretierbar. Sie funktionieren. Das zählt. Und dass sie gut sind, lässt sich daran überprüfen, dass bei ATLAS und CMS ähnliche Fragen gestellt werden und sie zu gleichen Ergebnisse durch unterschiedliche Herangehensweisen, Software, Algorithmen, sogar Programmiersprachen - C++ und Python gelangen. Sie tun ihren Job.“ Sebastian sieht das im Gegensatz zu vielen anderen nicht so. Er meint: „Natürlich wissen wir, wie das da funktioniert. Die Neuronen und Parameter haben wir ja selbst hineingegeben, die sind ja nicht im Area 52 vom irgendwelchen Aliens hinterlassen worden. Jeder Punkt hat eine Aktivierungsfunktion - und die ist bekannt." Die LHCExperimente sind so komplex, dass für die meisten Physiker_innen weite Teile der Apparatur zur Black Box wird. Die simulierten Daten erscheinen ihnen im Alltag nicht, wie von Merz beschrieben als „Antwortmaschinen“, die sich in "Fragemaschinen“ umwandeln (2002: 280), sondern werden unter dem Blickwinkeln der Praktikabilität und Funktionalität behandelt, fast schon mit einer post-positivistischen Resigniertheit als das akzeptiert, 
was sie über Jahrzehnte hinweg geworden sind: Verlässliche Größen, an die man glauben muß, und an deren Glaube man am Besten nach dem Feierabend zweifeln darf und sollte, weil es das perfekte Experiment per definitionem nicht geben und man nach ihm nur streben kann.

Die Konkurrenz schläft nie, erst recht nicht in den Seamful Spaces, wo rund um die Uhr gearbeitet wird. „Wenn ich morgens aufwache und in meine Emails schaue ist oft eine Arbeit vorangegangen, dann weiß ich, dass während ich geschlafen habe, auf der anderen Seite der Welt weitergearbeitet wurde“, sagt Sebastian. In den Meetings, insbesondere aber in den Approval Meetings und Status Reports taucht immer die Frage auf, wo ATLAS oder im umgekehrten Fall CMS gerade steht und wie die Qualität des Projekts im Vergleich mit der Konkurrenz abschneidet. Durch die Installation des "gleichen Anderen“ wird die Arbeitsleistung an der Performance der gegnerischen Gruppe ablesbar. Eine leitende Managerin von ATLAS sagt im Interview: „Deshalb ist das Verraten von Forschungsergebnissen so ein Problem für uns. Es ist einfach unfair und unethisch. Wie betrügen beim Spiel." Das Deep Play der Wissenschaft kennt nur ein Ziel: "Wahrheit" - bewusst in Anführungsstrichen gesetzt, denn sie schließt das Wissen um ihre mögliche Vergänglichkeit mit ein. Wer versucht Abkürzungen zu nehmen, gefährdet nicht bloß seinen eigenen Status, sondern hier in diesem Fall das Ansehen, Wohl und Wehe ganzer Kollaborationen.

So verwandelt sich die von Wissensaustausch und Informationspolitik geprägte Arbeitsorganisation in einen Playground, auf dem sich Menschen treffen und ebenso produktiv wie destruktiv, gleichsam eu- wie dysagonal miteinander arbeiten. Wie beim Deep Play in Bali, wo die Hähne stellvertretend für ihre Besitzer und für ganze Familien miteinander kämpfen, treten am CERN Menschen in den Arbeitsring, die symbolisch das Ideal der massenbasierten Bestengesellschaft verkörpern und dabei ebenso oft Verletzungen erleiden, wie sie Erfolg haben. Die Ungleichheiten werden zwar in einer Trading Zone ausgehandelt, aber diese Zone gleicht, sobald man sich die Arbeit in den Gruppen anschaut in der Praxis einem Playground auf dem es, wie auf jedem Spielplatz, alle möglichen Figuren gibt, vom Alphatier über den Blender, den Klassenclown bis hin zum Außenseiter. Welche Position die Spieler haben, wie lange er oder sie schon dabei ist, bleibt entscheidend, selbst wenn alle im gleichen Sandkasten sitzen und nach den gleichen Regeln spielen. „Eigentlich ist hier Platz für jeden“, sagt Valentina, „auch wenn es manchmal schwierig ist, bestimmte Leute unterzubringen. Abgesehen vom Vollkoffer, der mit niemandem spielen kann, haben wir hier eigentlich für jeden etwas zu tun, wenn er gut ist.“

Die meisten Aufgaben stellen modulare Bestandteile einer größeren Gruppenarbeit dar und setzen sich aus verschiedenen Tätigkeiten zusammen: Zunächst einmal sind Internet Communication Technologies (ICTs) 
anzuwenden, um alltägliche Korrespondenzen per Email sowie insbesondere via Video und Skype zu erledigen, die oftmals verlangen Arbeiten einer Gruppe zu koordinieren. Ein weiterer Bestandteil bildet insbesondere bei Doktorand_innen das Programmieren (alleine oder gemeinsam mit anderen). Die erfahreneren Physiker_innen koordinieren Gruppen, managen die Analysen oder die Entwicklung von Software, ordnen dabei die Arbeit von anderen und bringen im Fall von Aufsätzen etwa das Gemeinschaftswerk in die richtige Form, damit die Ergebnisse in den Meetings produktiv diskutiert werden können. Eine wichtige Aufgabe in der informationsbasierten Arbeit der experimentellen Hochenergiephysik besteht darin, die kollektive Arbeit von Physiker_innen an Codes zu koordinieren. Zu diesem Zweck werden Logistik-Tools eingesetzt, die es ermöglichen, die Arbeit zu vereinheitlichen oder Aufgaben zu verteilen. Alle paar Jahre wieder wird dabei das Codemanagement grundlegend überarbeitet. Bei ATLAS wurde vor Kurzem von svn (Subversion) auf Git umgestellt. Git vereinigt die Arbeitsleistung von vielen Menschen, die gemeinsam an Codes arbeiten. Durch das Ticketsystem JIRA, einem Issue Tracker werden die Gruppenarbeiter über laufende Arbeitsprozesse informiert. Bei den Softwareentwicklungen wird auch Jenkins eingesetzt, eine Anwendung, die plattformübergreifend, "continuous integration and continuous delivery" ermöglicht und somit das Bauen und Testen von Softwareprojekten erleichtert, bevor die Codes "gemerged“, also miteinander verwoben werden. ${ }^{11}$ Dabei kommt es immer wieder zu Fehlschlägen. Gregor kommentiert aus seiner Erfahrung: „Fehlgeschlagene Tests sind Teil des Workflows. Jeder macht Fehler und niemand muss sich schämen, wenn ein Test fehlschlägt." Ein Nebeneffekt von Git ist, dass alle Gruppenmitglieder über die (Fehl-)Arbeiten der anderen informiert sind. Nach einem Merge, werden die vorherigen Arbeitsschritte, die „Geschichte“ des Codes und der Veränderungen gelöscht. „Die Geschichte wird umgeschrieben“, sagt Sebastian, der sich mühselig in die neue Software einarbeitet und an den neuen Arbeitsstil mit seinen Atomic Commits, das heißt sehr kleinen Tasks, die nun in alle Verzweigungen des Algorithmus übertragen werden können, erst noch gewöhnen muss: „Ich neige oft dazu, Monster Branches zu machen, das heißt ich ändere 50 Files, benenne Kategorien um und Merge das alles auf einen Schlag in den Master Branch, was dann zu Problemen für die anderen führt, die erst einmal wieder gelöst werden müssen“, gesteht er selbstironisch. Die Doktorandin Marlene erklärt: „Wenn einer in 50 Files eingreift, ist die Wahrscheinlichkeit einfach groß, dass die anderen an ähnlichen Files gearbeitet haben und Konflikte bekommen, die aufgelöst werden müssen." Bei den vorher eingesetzten Plattformen war die Arbeitsweise linearer. Sie führt aus: „Jetzt wird die Wahrheit umgeschrieben, weil man durch das Mergen einen Rebase macht, das heißt in allen Branches die Änderungen durch Git vornehmen 
lässt und die Konflikte, die man bei sich ausgelöst hat, klärt.“ Die Geschichte erscheint immer unwichtiger, die Praxis immer verwobener, sobald die Maschinen rechnen. Hauptsache das Programm läuft fehlerfrei und die an hermeneutische Verfahren erinnernde algorithmische Rekonstruktion der Ereignisse funktioniert (Dippel \& Mairhofer 2017: 83-86).

Einige der älteren Physiker_innen beschweren sich über das neue Codemanagement, das sie nicht verwenden können, was „vielleicht nur den Jungen nützt, die später bei Google arbeiten." Die meisten versuchen eher mühselig auf dem neuesten Stand zu bleiben. „Ich verstehe oft nicht einmal mehr, was die Jungen sagen“, beschwert sich ein älterer Physiker, „obwohl ich früher besonders im Programmieren drinnen war, solange wir noch Subversion hatten. Mit Git wird alles schwieriger, weil lauter Begriffe verwendet werden, die ich einfach nicht kenne." Sebastian findet jedoch: „Du musst schon immer am Ball bleiben, wir Älteren haben uns an die junge Generation anzupassen, nicht jeder wird schließlich später hier arbeiten können und braucht dann die Tools draußen." Dass es für die erfahreneren Physiker_Innen nicht leicht ist, bringt die Jungen zuweilen zum Tratschen und gibt ihnen Selbstbewusstsein: „Die Älteren tun sich etwas schwer mit der Umstellung auf Git," findet auch die Dotorandin Marlene und schmunzelt dabei über ihren Gruppenleiter, der sich noch an die neue Form der Dokumentierung gewöhnen muss und gerne mit Jenkins testet, anstelle lokale Tests vorzunehmen, wodurch dann alle Teilnehmer_innen zwei Emails erhalten, was zu Verdruss führt. Testsuites nehmen Zeit in Anspruch und verlangsamen die Arbeit der gesamten Gruppe. „Nur Bugs suchen dauert länger“, sagt Marlene, „die Sicherheit geht vor.“ Gregor überlegt: „Meine Erfahrung ist: lokal testet man das, was man gerade selbst entwickelt, bzw. verbessert hat. Wenn das alles passed, dann commited man und online läuft dann die gesamte Testsuite die alles testet. Die checkt dann auch, ob meine Code-Änderungen anderes Zeug kaputt machen.“ - „Wenn jedes Mal ein Test fehlschlägt ist das nicht zielführend und kostet zu viel Zeit“, kommentiert Robert lakonisch, ein Doktorand der ebenfalls in der gleichen Gruppe arbeitet, über die Umstellungsschwierigkeiten seines älteren Gruppenleiters. An diesem Dialog lässt sich beobachten, dass Anglizismen am CERN im Deutschen, ebenso wie in vielen anderen Sprachen, die hier gesprochen werden, selbstverständlich sind - das Alltagsenglisch erscheint nicht als Kreol, wie von Peter Galison vorgeschlagen, sondern als ein Fachdialekt, der von Termini geprägt ist, die sich durch das jeweilige Experiment ergeben, der sich mit jedem neuen Programm entwickelt und sogar von Generation zu Generation ändert.

Oft tauschen sich die Gruppenmitglieder über die Funktionsweise der Plattformen und Anwendungen so aus, als ob es sich dabei um lebende Wesen handelt: „Jenkins loggt sich auf GitLab ein“, sagt Robert. Er hat agen- 
cy. Einmal fragt Marlene: „Der Jenkins kompiliert schon den kompletten Merge Request?“ Robert meint: „Uff, der hat einen Cache, wenn Jenkins merkt, dass sich da nichts geändert hat, wird es nicht neu kompiliert. Oder wie meinst du? Ich muss zugeben ich kenne mich damit eigentlich zu wenig aus um der Admin davon zu sein." Robert hat die Aufgabe gerade erst von einem anderen übernommen, der das CERN verlassen hat und inzwischen bei der Schweizer Post arbeitet. Marlene überlegt: „Naja, normalerweise sollte er, wenn man schon einmal einen Merge Request gemacht hat, wenn man dann neu pusht, automatisch neu kompilieren. Da hat es die letzten Wochen Probleme gegeben, aber ich glaube das geht jetzt wieder? Und sonst, wollte ich fragen, ob es eh so ist wie vorher, also wenn man zum ersten Mal einen Merge Request aufmacht, dass der komplette Merge Request kompiliert wird und nicht die Commits einzeln?" Gregor sagt daraufhin: „Also die Jenkins Settings sind jetzt so wie vorher. Es kamen die letzte Woche alle Jenkins-Nachrichten doppelt, deshalb hast du das vielleicht gedacht, weil der Jenkins-Server geklont war und beide Clone Tests laufen gelassen wurden und dann kommentiert haben. Und die Klone haben seltsamerweise um viele Stunden unterschiedlich lang gebraucht, um einen Job anzufangen. Ausführen war gleich schnell." Marlene fragt herausfordernd: „Welcher war schneller, deiner oder der Alte von John?" Der Agon hat kein Anfang und kein Ende. Robert meint: „Ich glaub mal so mal so. Heute kam eine Jenkins Message vom Server der schon seit Freitag keine Webhooks ${ }^{12}$ mehr bekommt." Und beide lachen über die Maschinen. Git wird von den Jüngeren als vorteilhaft beschrieben, weil es modernes Programmieren ausmache, man gemeinsam besser an Codes arbeitet und es die Kommunikation und Kollaboration erleichtert „auch wenn die Kontrolle des Einzelnen größer ist", meinen Gregor und Marlene unabhängig voneinander.

In dieser Passage lässt sich, neben der Anthropomorphisierung von Algorithmen und Tools, das hochspezialisierte und an den informatischen Gegenständen ausgerichtete Vokabular der Hochenergiephysiker_innen beispielhaft veranschaulichen. Die Sprache hat längst das Feld der Physik verlassen und liegt ganz im Bereich der Informatik. Während es für jüngere Physiker_innen kaum Probleme bereitet, von einem Plattformsystem ins nächste $\mathrm{zu}$ wechseln und sich neuen Verfahrensweisen anzupassen, erfordert diese Umstellung für Ältere eine längere Anpassungszeit, die zu Fehlern und Häme der untergebenen Jüngeren führen. „Jedes Jahr kommt etwas Anderes. Irgendwann wird der Aufwand neben dem Management einfach zu groß und man kommt nicht mehr so leicht mit, und doch muss man eben am Ball bleiben" sagt Sebastian. Die älteren Mitglieder der Kollaborationen müssen primär Konzepte umsetzen und entwerfen, garantieren die Produktivität in ihren Arbeitsgruppen ebenso wie die grö- 
ßeren Entwicklungen der Experimente. „Erst aus dem Zusammenspiel von Erfahrung der Älteren und aktuellen Techniken entsteht hier das Neue“ resümiert Marlene. „Trotzdem,“ sagt Johannes als erfahrener Gruppenleiter, „halte ich mich auf dem Laufenden, was das Coden anbelangt. Wenn Du nicht hands on bist und mit einem Fuß in der Tür bleibst, verpasst Du den Anschluss und verstehst nicht mehr, was Deine Gruppe macht."

Auch wenn sich aufgrund unterschiedlicher Wissensbestände, Erfahrungswerte und Aufgabenbereiche eine Hierarchie ergibt, betonen die Mitarbeiter_innen am CERN immer wieder die im Vergleich zu anderen großen Forschungseinrichtungen, wie etwa Max-Planck-Instituten oder klassischen Lehrstühlen deutlich flachere Hierarchie vor Ort. „Mir ist es egal, ob ich mit einem Professor rede, oder mit einem wissenschaftlichen Mitarbeiter, Hauptsache die Physik stimmt. Den meisten ist das egal,“ sagt ein Gruppenleiter - und fügt hinzu: „Wenn sie gut sind.“ Wie die Physikerin Corinne Pralavorio in ihrem CERN-Blog schreibt: „Die Leiter des Experiments, als ,Spokespersons' bekannt, sind in Wirklichkeit keine Line Manager weil sie hier für ein Institut arbeiten und Rechenschaft ablegen müssen (Pralavorio 2016, Übs. d. A.)." Macht und Einfluss in der Kollaboration zeigt sich also weniger durch klare Befehlsstrukturen und Positionen im Arbeitsgefüge, als durch innere Vernetzung, Informationsvorsprung und die „Kürze des Drahts“ im Kontakt mit einflussreichen Mitgliedern der Kollaboration, die öffentliche Positionen wie etwa die Leitung eines Experiments oder ähnliche administrative Aufgaben bekleiden: „Wenn ich eine E-Mail an Fabiola [Fabiola Gianotti, die Generaldirektorin des CERN; Anm. d. A.] schreibe, dann antwortet sie mir noch am gleichen Tag“, sagt Sebastian und gibt damit seinen Kolleg_innen am Mittagstisch zum Ausdruck, dass er einen schnelleren Weg zu einflussreichen Figuren besitzt und wichtige Meetings über ihn laufen, Hierarchien also im Umkehrschluss doch eine zentrale Bedeutung besitzen.

Physiker_innen am CERN spielen in Teams, wobei sie die Arbeit anderer auf die Probe stellen müssen, weil sie wissen, dass auch sie einen Fehler machen können. Niemand würde von sich behaupten, er habe nie einen Bug in ein System gesetzt. Das Wissen um die eigenen Fehlerhaftigkeit und die Fragilität des Experiments selbst verstärken somit das wechselseitige Überwachen und die Notwendigkeit immer mit anderen $\mathrm{zu}$ interferieren. Die Experimente sind viel zu komplex, als dass man Experte aller Gebiete sein könnte, immer verändert sich irgendwo der Wissensstand und stets muss daher auch ein „Update“ mit einkalkuliert werden, was die Fachgespräche auf den Gängen vervielfacht.

In diesem Comic sieht man viele bisher angedeutete Aspekte verdichtet in drei Bildern (Abb. 2). Einerseits wird deutlich, dass die hierarchische Organisation gerade leitenden Figuren oft den Spielraum gibt, Arbeit, die 


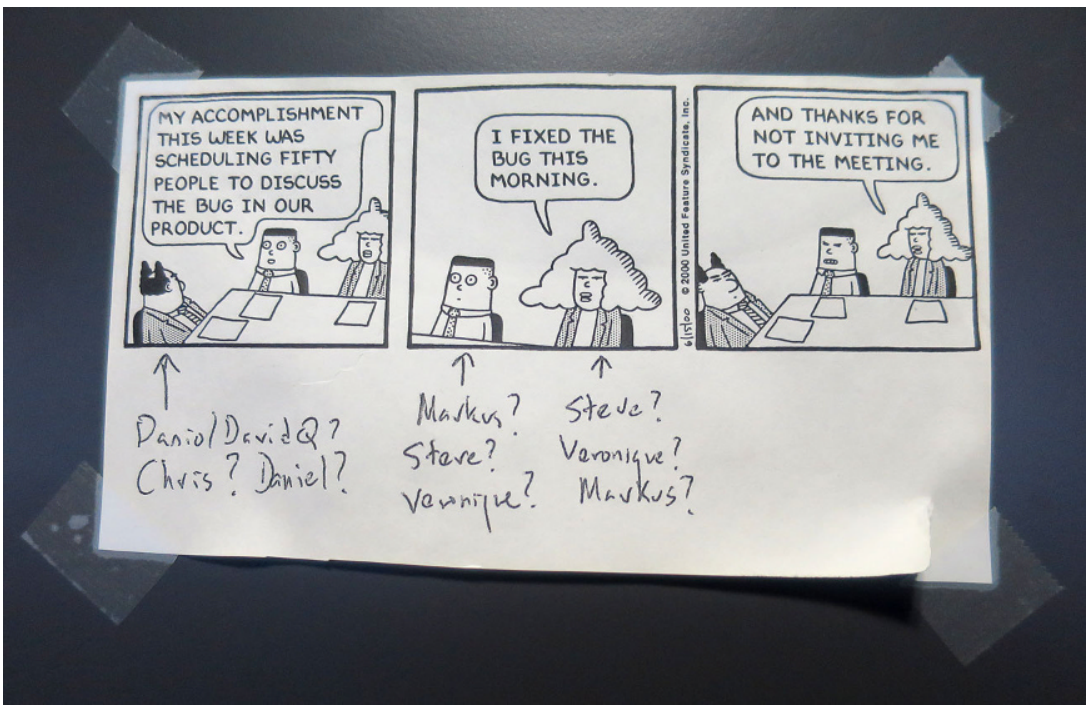

Abb. 2 Bugs fixen (Foto A. Dippel)

andere erledigt haben, als die ihre auszugeben. Deutlich wird auch der Druck, der von der Führungsebene auf die einzelnen Teilbereiche der Experimente ausgeübt wird. Am Ende des Tages geht es vor allem darum, dass die "Sache läuft“. Wie es geschieht, wer es macht, ist dabei zweitrangig. Und nicht zuletzt wird in diesem Comic auch die stets gegenwärtige Ungerechtigkeit in solchen Kollaborationen thematisiert. Dabei verweist der Comic selbst auch auf existierende Geschlechterhierarchien, die im Alltag oft dazu zu führen scheinen, dass gerade Frauen besonders viel arbeiten, ihre Arbeit aber weniger Anerkennung erfährt, sondern oft von einem männlichen Kollegen sogar für sich vereinnahmt wird. Wer durchsetzungsfähig ist, muss am CERN seine Qualitäten ausspielen, denn nur so ist eine langfristige Position garantiert. Deshalb spielen viele mit „schmutzigen Karten“, wie ein Cernie erzählt, der frustriert von einigen seiner Kollegen resümiert: „Das CERN ist ein Schlangennest. Und wer besonders pushy ist, der wird hier überleben und hat größere Chancen eine Dauerstelle im Management zu bekommen. Manche sind so pushy, sie pushen in alle Richtungen. Wie ein Esel. Sie haben keine Ahnung, aber Einfluss. Und wir müssen dann versuchen alles zu retten. Achte mal darauf, ob die CERN-Dauerstellen nicht insbesondere von solchen Management-Typen besetzt werden, die dann vor allem Macht ausüben und Druck in der Kollaboration aufbauen, während die meisten Forscher_innen auf den schlechtbezahlten Dauerstellen der Nutzeruniversitäten sitzen." Wann immer solche Aussagen von Mitgliedern der Kollaborationen kommen und 
ich daraufhin nachfrage, ob sie nicht selbst der Meinung seien, dass hier auf hohem Niveau gejammert wird, reagieren alle sofort mit nachdrücklichem Kopfnicken. Und geben gleich zu bedenken, dass die Funktionsweise der Organisation überragend sei, doch die Experimente und vor allem auch die Datenanalyse noch „besser“ funktionieren könnten, wenn einiges hier eben „professioneller“ geregelt und das Human Resource Management nicht zu viele bürokratische Regulierungen in die Kollaborationen implementieren würde. Johannes meint: „Die HR [Human Resource Abteilung] hat in den letzten Jahren einfach zu viel Einfluss bekommen. Das Department wächst die ganze Zeit und damit fällt ihnen auch immer mehr ein, wie sie uns regulieren können." Deep Play im Neoliberalismus trägt hier selbstverständlich bürokratische Züge und selbst die Open Data und Open Platform Policy des CERN fügt sich trotz bester Absichten in das gegenwärtige Paradigma.

Wissenschaftliches Arbeiten verlangt nach Neugierde und Determiniertheit. Durch die steile Lernkurve sind Kollaboration und Konkurrenz gekoppelt: „Wenn Du am CERN ankommst, dann sagen sie Dir: ,Weißt Du, die Meisten lernen hier $\mathrm{C}++$ in sechs Wochen. Du wirst es in drei schaffen. Du bist besser, ich weiß es." Von der Führungsebene her besehen ist so eine Aussage als Ansporn gedacht, der im besten Fall aus dem Team „mehr als einen Monat Arbeitsleistung herausholt, die sie bei geringerer Forderung nicht erfüllt" hätten, erfahre ich von einem Physiker aus einer Higgs-Analysegruppe.

Von dem Moment an, wo Physiker_innen sich in den if-then-whileGrammatiken des Programmierens bewegen können, sind sie dazu gezwungen, sich vorzustellen was sie zu programmieren haben, müssen sie über Dinge sprechen, die nicht visualisier-, nur kodierbar sind, um mathematische Hypothesen zu testen. Und damit hat das Spiel der Erkenntnis begonnen, das eben immer auch ein sozialer Agon ist, gerade bei der Entwicklung von evolutionären Algorithmen zur Analyse von Big Data.

\section{Ludische Plattformen: Epistemische Variation}

In diesem Abschnitt werden systematische Auslagerungen von Forschungsproblemen auf ludische Plattformen beschrieben, die spezifisch durch den Umgang mit Big Data entstehen und durch Spiele gelöst werden können, indem wissenschaftliche Probleme in Challenges ausgelagert werden. Neben diesen Formen des Spiels dient das Spiel auch der Simulation und dem Begreifen von informatischen Problemen für die Physiker_innen selbst. So gibt es einen Playground für Experten, der sich in der von Google entwickelten Open Source Software Library TensorFlow befindet und vermittelt, 


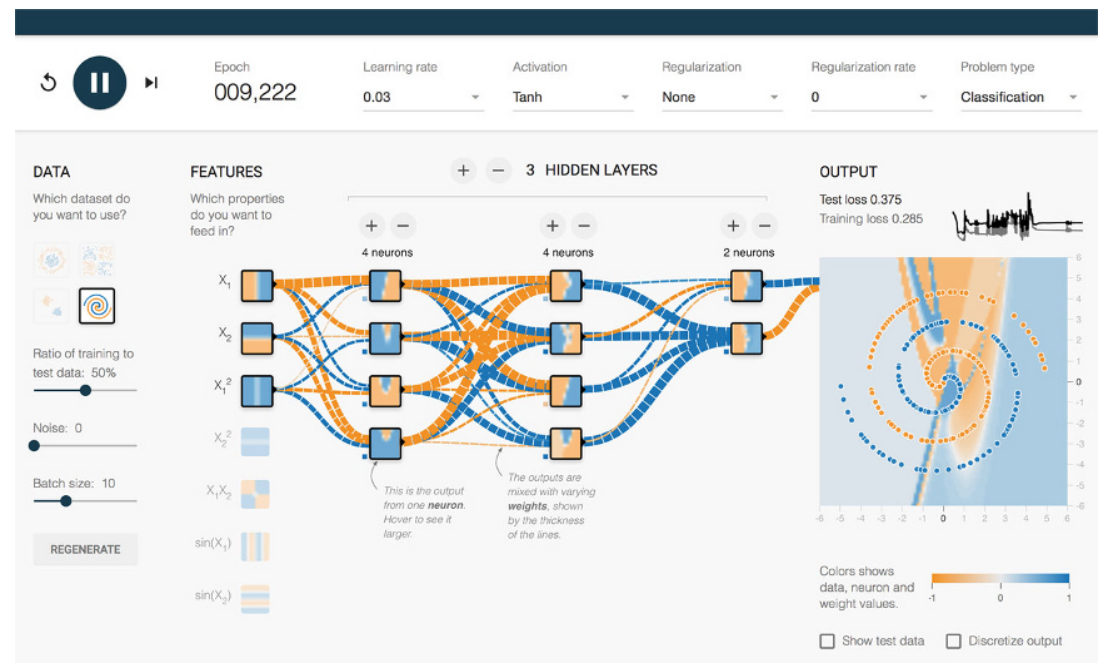

Abb. 3 Playground, Tensor Flow Platform (Foto A. Dippel)

wie neuronale Netze funktionieren (Abb. 3). Er wirbt mit dem Slogan: "Tinker with a Neural Network Right here in your Browser. ${ }^{\text {"13 }}$ Hier konvergieren Name und Praxis physikalischen Arbeitens in Zeiten von Big Data vollends.

Verbreiteter sind Spiele als Werkzeuge, die unter der Bezeichnung Citizen Science Games oder Online Crowdsourcing Games und Expert Challenges bekannt sind. Ihr Ziel ist vor allem die Verbesserung der neuronalen Netze, und dient den Machine Learning Algorithmen. Big Data-spezifische Probleme werden gelöst, indem Code und Daten online gestellt, Teams zu Wettbewerben aufgerufen werden, um die hier anfallenden Probleme zu lösen. Challenges bilden inzwischen integralen Bestandteil des an Big Data orientierten Forschungsprozesses. Die von Hintergrundrauschen und Fake Tracks übersättigten Proton-Proton-Experimente des CERN stellen eine große Herausforderung für algorithmische Mustererkennung dar. Um Algorithmen zu entwickeln, die mit dieser Menge an Big Data umgehen können, werden Datensets in zwei Teile gesplittet, wobei ein Teil dazu dient, Algorithmen zu trainieren und der andere dazu dient, Algorithmen zu testen. Die Rechenleistung dieser Algorithmen ist hoch, die Datenmengen sind so groß, dass sie nicht auf einem Computer berechnet werden können, weshalb ein ganzes Netz von Computern im LHC-Grid parallelgeschaltet werden muss, in denen Algorithmen miteinander verschmolzen werden, damit das beste Modell zur Vorhersage eines Ereignisses entwickelt werden kann. Es setzt sich aus verschiedenen Algorithmen zusammen und emergiert evolutionär in einem Netzwerk. Die erforderlichen evolu- 
tionären Machine Learning Algorithmen stellen eine Grenze der Arbeit für viele Physiker_innen dar: Sie sind schließlich keine Informatiker_innen, sondern bloß Laien, wie sie selbst sagen. Christian meint: „An anderen Orten wird sehr viel professioneller programmiert als bei uns. Das frustriert mich manchmal schon." Um die Mustererkennung und Modellbeschreibungen zu verbessern, setzen die Physiker_innen in den Großkollaborationen ATLAS und CMS daher auch Expert Challenges ein, bei denen Teams an Algorithmen arbeiten, die eine valide digitale Mustererkennung aus der Big Data Masse überhaupt erst ermöglichen. Generelle künstliche Intelligenzen sind für unterschiedlichste Datensets einzusetzen, auch wenn es viele verschiedene „Familien“ von Machine Learning Algorithms gibt. Die Probleme, die am LHC bei der Mustererkennung ihrer Datenmassen anfällt, sind vergleichbar mit den Herausforderungen, die Firmen wie Facebook oder Google zu bewältigen haben und die zur Entwicklung neuraler Netzwerk-Intelligenzen wie „Alpha Go“ beitrugen, der 2016 den achtzehnmaligen Weltmeister Lee Sedol in dem komplexen Spiel Go geschlagen hat.

Die meisten Physiker_innen lernen erst am CERN, mit Python oder C++ zu arbeiten. Lange Zeit entwickelten Hochenergiephysiker_innen ihre Algorithmen für die bei den Experimenten am LHC anfallenden Algorithmen der so genannten MVA (Multi-Variable-Analysis) vollständig selbst. 2014 kam auf der Internetplattform Kaggle, die sich auf solche Programmierund Big-Data-Challenges spezialisiert hat, eine von ATLAS initiierte $M a-$ chine Learning Challenge zum Einsatz, die der Kollaboration helfen soll, in ihrem Gebiet eingesetzte generelle künstliche Intelligenz zu verbessern und dabei auf das Wissen von Informatiker_innen aus der ganzen Welt zurückgreift (Abb. 4). Für Teams, die sich an solchen Wettbewerben beteiligen, stellt der bereitgestellte Datensatz ein perfektes Übungsfeld im Umgang mit Big Data dar. Während die Physiker_innen also ihr Problem an Fachleute auslagern, werden im Gegenzug die selbstlernenden Algorithmen in der Mustererkennung verbessert. Das CERN spart dadurch Arbeitsleistung, die Teilnehmer der Challenges sammeln dabei Erfahrung und gewinnen symbolisches Kapital. Stolz erzählt mir etwa ein Informatiker vom MIT im Februar 2017, dass er mit einem CERN-Datensatz arbeitet, der in die Arbeit des CERN einfließen wird. Die erste Higgs Boson Machine Learning Challenge war indes von Mai bis September 2014 auf Kaggle ausgeschrieben. Das Spiel brachte 1.785 Teams, 1.942 individuelle Spieler und 35.772 Eingaben aus der gesamten Welt zusammen. Den Teilnehmer_innen war die Möglichkeit gegeben, mit einem Trainingsset von 250.000 Daten zu operieren und einem Testset von 550.000 Ereignissen zu arbeiten. ${ }^{14}$ Die größte Herausforderung, sagt David Rousseau, einer der Initiatoren, „war sicherzustellen dass der Reichtum an Ideen, 


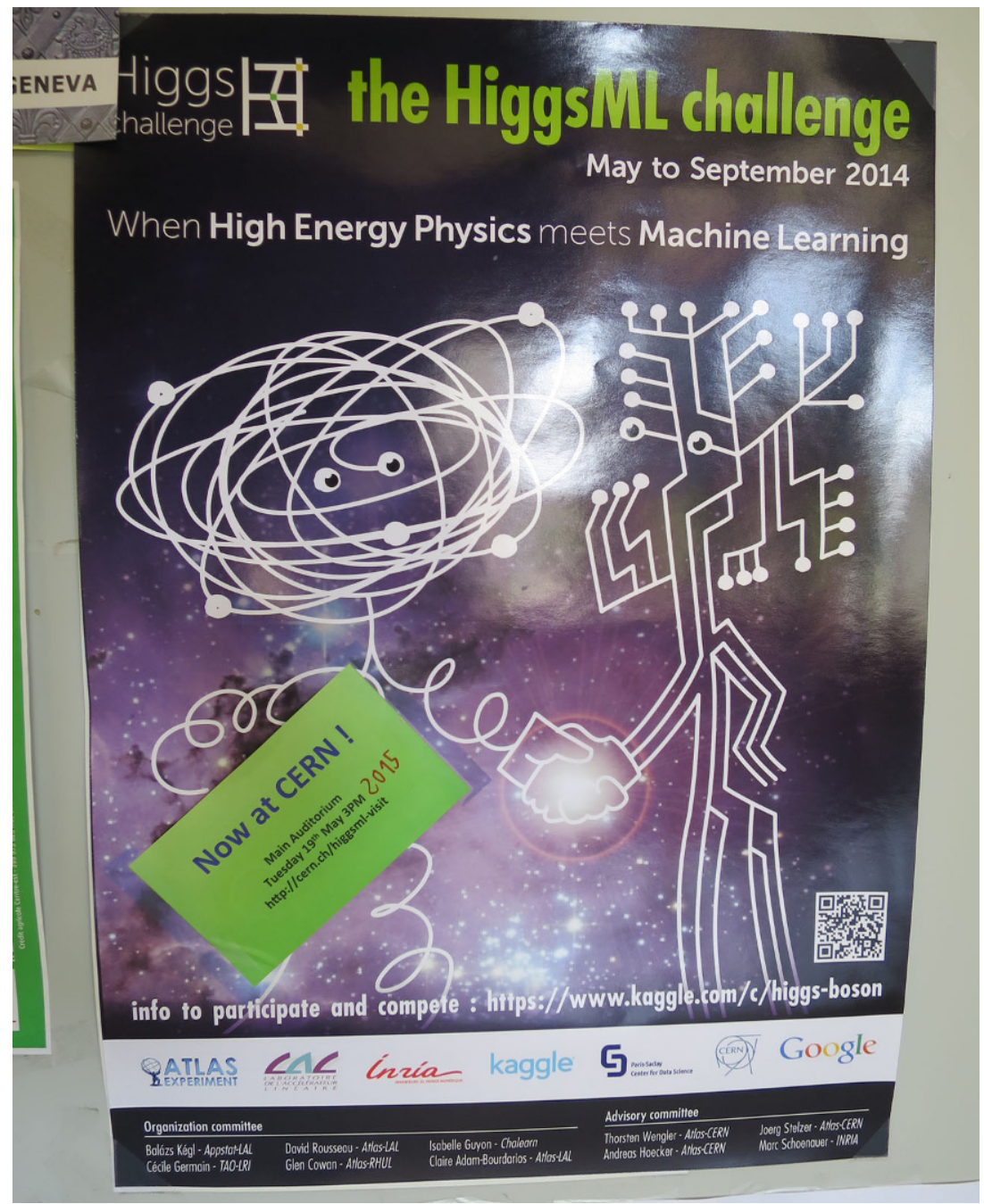

Abb. 4 HiggsML Challenge, Kaggle Platform

Software und Algorithmen, den die Teilnehmer_innen zusammengetragen haben, die Hochenergiephysik und den gelebten Physikalltag durchdringen." Neuere Challenges, wie eine aktuell erarbeitete Machine Learning Challenge von ATLAS, bei der mehr als eine Millionen Datenereignisse zur Verfügung gestellt werden, zeigen aber auch eine andere Seite des heiß umkämpften Marktes im Umgang mit Big Data und zeugen von dem hochkompetitiven Wettrennen, das von führenden Unternehmen ausgetragen wird. Johannes erklärt mir: „Es nehmen auch Teams von Google und Facebook oder aus dem Silicon Valley an der Challenge teil. Bei uns hat sich 
noch kein Team zurückgezogen, in Berkeley gab es mal eine Challenge, wo sich Facebook und Google einen Stellvertreterkrieg geliefert haben. Kurz vor Ende der Challenge haben sich die Teams aus dem Wettbewerb zurückgezogen. Es ist also nicht notwendigerweise der beste Code, der bei solchen Challenges gewinnt." Die Teams von Facebook oder Google ziehen sich deshalb aus Challenges zurück, weil ihr Code Teil des Betriebsgeheimnisses darstellt und in einer marktbasierten Wirtschaft eben keiner Öffentlichkeit zur Verfügung gestellt werden soll. Die Möglichkeit ihre generellen künstlichen Intelligenzen mithilfe Open Source gestellter Big Data zu verbessern, lassen sich die Teams des Silicon Valley ebenso wie wissenschaftliche Gruppen an Universitäten nicht entgehen. Als öffentliche Organisation ist das Wissen des CERN jedoch als ein Cosmopolitan Common (Disco \& Kranakis 2013), ein kosmopolitisches Allgemeingut angelegt und unterliegt damit spezifischen Lizenzregelungen, so etwa der GNU General Public License. In einer Welt, deren Kulturen sich überlagern wird Wissen medial vermittelt, weit über die staatlichen Interessen hinaus rechtlich geregelt, in deren Interessenrahmen der Ursprung dieser Allgemeingüter oftmals lag. ATLAS etwa hat sogar seinen Code als Open Source der Allgemeinheit zur Verfügung gestellt. Das CERN profitiert bei den Challenges von dem Einsatz der Citizen Scientists und Experten durch den Wissenstransfer. Auch wenn die Implementierung der Algorithmen selbst wiederum viel Arbeit bereitet, fließen die Ergebnisse der Outsourcing Games in die Mustererkennung am CERN ein.

Online-Citizen-Science-Spiele wie das auf menschliche Mustererkennung ausgerichtete Spiel Higgs Hunters (2015), bei dem die Spieler die vorhandenen künstlichen Intelligenzen verbessern, und oben beschriebene Expert Challenges, die für die Verbesserung und Erneuerung von Algorithmen eingesetzt werden, sind gerade deshalb im Umgang mit Big Data so erfolgreich, weil sie die „produktiven Handlungen von miteinander verbundenem menschlichem Geist" (Terranova 2013: 33; Übs. d. A.) ermöglichen (Abb. 5). Während die Expert Challenges von den Teilnehmer_innen hohe Programmierkenntnisse vorrausetzen, stellt das von den Universitäten Oxford, Birmingham und der New York University erarbeitete und auf der Plattform Zooniverse bereitgestellte Higgs Hunters das erste Spiel dar, das für eine breitere Öffentlichkeit ausgerichtet ist. Es spekuliert darauf, dass Laien ihre Freizeit am Computer zur Verfügung stellen, damit Proton-Proton-Kollisionen simuliert werden können. Bis Oktober 2016 haben sich insgesamt 32.288 Citizen Scientists an dem Spiel beteiligt, wobei die meisten nur "eine Hand voll“ von Bildern klassifizierten, während zehn Teilnehmer_innen je 5.000 Bilder und ein besonders „hingebungsvoller“ Teilnehmer 20.000 Bilder klassifizierte. In allen Fällen waren sie ebenso stark wie die Algorithmen von ATLAS und in einigen Fällen übertrafen sie 


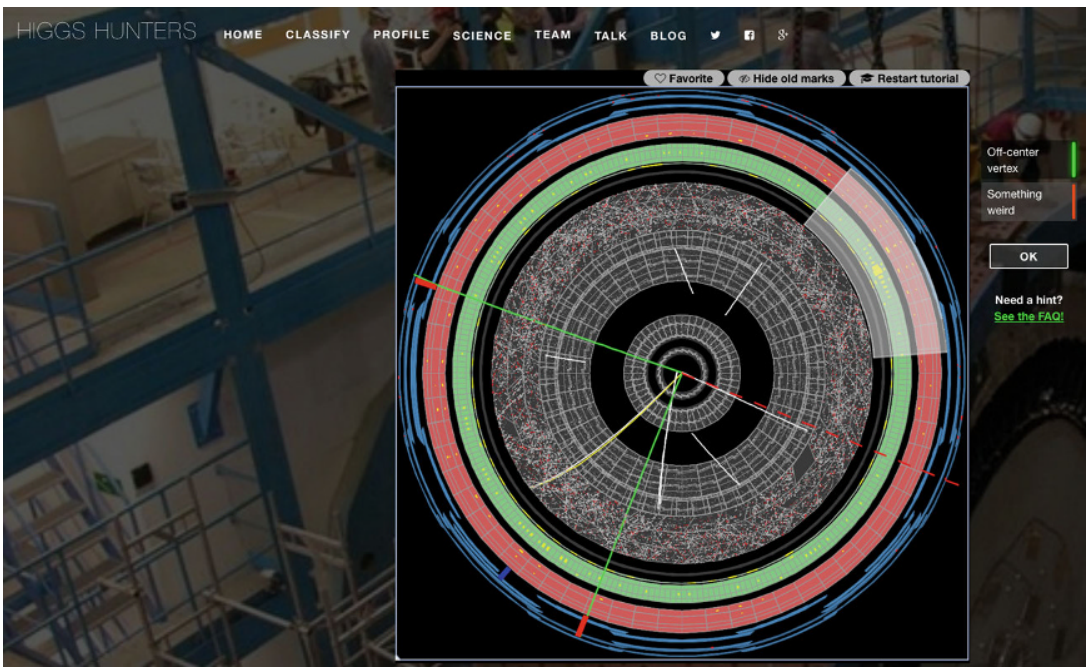

Abb. 5 Higgs Hunters, ein Online-Citizen-Science-Projekt das auf der Zooniverse-Plattform angeboten wird

sogar die digitalen Rekonstruktionen in der Mustererkennung (Barr et al. 2016: 8-9).

Im Wissensspiel der Citizen Science Games gewinnen alle: Die Spieler_innen tragen auf ihre Art zur Wissensökonomie des CERN bei. Als Citizen Scientists haben sie die Möglichkeit am „highest profile scientific project of our time“ teilzuhaben (Barr et al. 2016: 1). Als Spielexperten können sie ihre eigene Stärke testen und wenn sie nicht durch Schweigepflicht gebunden sind, fließt ihre Arbeit in selbstlernende Algorithmen, die in den Detektorkollaborationen für die Auswertung und Simulation von Daten genutzt werden, mit ein. Während also die individuelle Motivation bei Spieler_innen, die sich an Citizen Science beteiligen von idealistischen Zügen und der Lust an einem Spiel mitzumachen geprägt ist, das außerhalb seiner selbst noch ein weiteres Ziel in sich trägt, profitieren die Experten in den Challenges und die Cernies selbst von Big Data, weil das Spiel in diesem Fall eine konkrete Verbesserung künstlicher Intelligenzen bewirkt. Die Wissenschaftler_innen erkennen die Bemühungen der Expert Science Community an und laden die Gewinner_innen dazu ein, an CERN-Workshops teilzunehmen, damit ihr Wissen in den Kollaborationen überhaupt aktiv wirksam werden kann. Die Ergebnisse der Spieler_innen finden darüber hinaus in akademischen Veröffentlichungen Anerkennung. Und nicht zuletzt profitiert langfristig die Forschung an der generellen künstlichen Intelligenz von diesem Deal. Die Trading Zone hat diese Plattformen und Open-Data-Portale des CERN am offenkundigsten in einen Playground verwandelt, auf dem Menschen und Algorithmen zu Playbouring Cyborgs 
(Dippel \& Fizek 2017) verschmelzen, um Wissen herzustellen und zum „Produser" (Kasperowski \& Hilman 2017) werden.

\section{Zurück auf Los}

Im Jahr 1989 wurde das World Wide Web Protokoll am CERN entwickelt (Gillies 2000). Heute tragen Netzwerkstrukturen wie das hier verwendete Cloud-System Grid, die Open Data Policy oder die eingesetzten evolutionären Machine Learning Algorithms zur Weiterentwicklung informationstechnologischer Innovationen bei. Big Data trägt zur Popularität der Forschungsinstitution bei. Mitglieder der Vorzeigekollaborationen ATLAS (A Toroidal LHC Apapratus) und CMS (Compact Muon Solenoid) eine „Assemblage“ von Menschen, Daten, Maschinen und Plattformen (Deleuze \& Guattari 1987: 4, 8, 23; Collier \& Ong 2008), an der sich die Frage nach dem Menschen ebenso stellen lässt, wie der Einfluss von neuen Technologien auf wissenschaftliche Forschungspraxen. Das CERN ist zum Deep Play geworden, in dem sich eine technologiebasierte Kultur wiederspiegelt und ihre Werte repräsentativ ausgehandelt werden, von der symbolischen Konzeptualisierung über wettbewerbsorientierte Arbeits- bis hin zu Plattformstrukturen, die Datenmassen verarbeiten.

Hier lässt sich beobachten, wie die von Donna Haraway beschriebenen „Grenzkriege“ zwischen Organismen und Maschinen (Haraway 1991) verantwortungsvoll ausgehandelt werden - indem sie in eine ludische digitale Infrastruktur eingebettet sind, wo Arbeit und Spiel miteinander fusionieren. Die „Wissensfabrik“ (Knorr-Cetina 1984) wird zu einem ludischen Grund, die Trading Zone verschränkt sich durch digitale Infrastruktur in Seamful Spaces zu einem Playground, ökonomisches Argumentieren trägt spielerische Züge. So entstehen Boundary Objects wie etwa (simulierte) Teilchen durch mediale Infrastrukturen und „socio-technical imaginaries“, halten „partizipatorische“ technologische Systeme (Shrum et. al. 2007) für eine Wissensgemeinschaft zusammen, deren spezifische Signatur durch sie mit entsteht. Spiel wird hier Dynamik der Reflexion und Modus der Relaxion, tariert das Wechselspiel von Kollaboration und Wettbewerb zum Zweck der Erkenntnis aus und gibt dem Zufall Raum.

Welche spezifischen historischen Umstände und kulturellen Besonderheiten dazu geführt haben, dass die Hochenergiephysik zur Modellwissenschaft für kollaboratives Handeln werden konnte und infolgedessen Kontrolle und Spiel so eine prominente Rolle in dieser Wissenschaft einnehmen, möchte ich an anderer Stelle genauer untersuchen. Ein zu befragendes Desiderat bleibt auch, welche Position die Big Data-orientierte 
Grundlagenforschung mit ihrer Ausbildung tausender Arbeitskräfte für die freie Wirtschaft und insbesondere das digitalisierte Marktwesen, die Datenanalyse und das Silicon Valley besitzt; welche Verknüpfungen sich also zwischen scheinbar ethisch freier und interessenloser Forschung mit einem neoliberalen Wirtschaftssystem herstellen lassen.

Das Ludische muss als eines der zentralen Charakteristika Big Dataorientierter Wissenschaft begriffen werden. Wenn die Natur nachgeahmt und auf einer Bühne zur Schau gestellt wird, spielen Menschen: Sie modellieren theoretische Kalkulationen, üben sich in logischem Argumentieren und freiem Assoziieren, suchen nach algorithmischen Lösungen im Bereich der Informatik und rechnen auf den „unendlichen Spielfeldern“ der Mathematik, spielen und dealen in ludischen Infrastrukturen mit Big Data. Physik in Zeiten von Big Data ist dazu verpflichtet, vertrauenswürdige, experimentell falsifizierbare Wahrheit zu erzeugen. Der Komplexitätsgrad führt zu spielförmigen Strategien, um gemeinsam Wissen zu produzieren. Ob systematisch eingesetzt oder durch unvorhergesehene Selbstorganisation hervorgerufen, induziert Spiel eine produktive Dynamik, befreit von strikter Rationalität und Funktionalität und verstärkt die Leistung wissenschaftlicher Kollaboration. Sei es bei der Arbeit oder in den Pausen: Wo mit großen Datenmengen Träume der Menschheit gesponnen und kulturell Natur modelliert wird, ist Spiel am Werk.

\section{Danksagung}

Mein spezieller Dank gilt Sonia Fizek und Ursula Rao für hilfreiche Kommentare zu einer früheren Version dieses Aufsatzes. Des Weiteren möchte ich Markus Rautzenberg danken, der mir durch frühen Einblick in meine Forschungsdaten half, das Verhältnis von Komplexität in Arbeitsprozessen und Spielförmigkeit zu durchdenken. Nicht zuletzt möchte ich mich für die Gastfreundschaft von David Kaiser bedanken, der mich während meines Aufenthalts als Visiting Assistant Professor im STS Department am MIT auf die inhärenten Machtkonstellationen in Kreolsprachen aufmerksam machte, ein Umstand, der schon in seiner Zeit als Doktorand am History of Science Department in Harvard im Blick auf das von Peter Galison entwickelte Konzept der Trading Zone diskutiert wurde und ein wichtiges Argument für die Entwicklung des Playgrounds darstellt. 


\section{Anmerkungen}

1 http://atlas.cern/discover/detector/trigger-daq (03.10.2017).

2 https://home.cern/about/computing (03.10.2017).

3 https://phys.org/news/2017-07-cern-centre-petabyte-milestone.html (03.10.2017).

4 http://opendata.cern.ch (03.10.2017).

5 Um die Persönlichkeitsrechte der Informant_innen zu achten werden für alle Mitglieder des CERN Pseudonyme verwendet und Situationen so dargestellt, dass nicht erkennbar ist, um wen es sich dabei handelt. Ich möchte mich an dieser Stelle bei meinem Feld herzlich für das Vertrauen bedanken.

6 Produktive Erkenntnisse über den Begriff Synagonismus wird in Zukunft aus dem DFGNetzwerk „Synagonismus in den Bildenden Künsten“ zu erwarten sein, an dem Yannis Hadjinicolaou, Joris van Gastel, Markus Rath, Lena Bader, Jasmin Mersmann und Maurice Saß mitwirken (https://www.kulturtechnik.hu-berlin.de/de/content/erfolgreicherantrag-fur-das-dfg-netzwerk-synagonismus-den-bildenden-kunsten/, 15.09.2017).

7 Ein Nash-Equilibrium ist ein Terminus aus der Spieltheorie, der rationale Entscheidungen für Konflikt- und kooperative Situationen favorisiert, während die Strategien aller Spieler individualisiert und verdeckt werden.

8 ATLAS (A Toroidal LHC AparatuS) ist - zusammen mit CMS (Compact Muon Solenoid) - einer von zwei general-purpose detectors am CERN, der ein weites Spektrum von Physik untersucht, von der Suche nach dem Higgs-Boson über Extra-Dimensionen und Teilchen, sowie Ereignissen, die Rückschlüsse auf dunkle Materie liefern.

9 https://press.cern/backgrounders/cern-answers-queries-social-media (03.10.2017).

10 Sternstunde Philosophie mit Harald Lesch, SRF 1, 09.09.2014. (http://www.srf.ch/ sendungen/sternstunde-philosophie/60-jahre-cern-harald-lesch-ueber-die-raetselder-physik, 03.10.2017).

11 https://wiki.jenkins.io/display/JENKINS/Meet+Jenkins (03.10.2017).

12 Webhooks erteilen über eine Server-Software die Nachricht über den Eintritt eines Ereignisses, und können eine Reaktion auslösen.

13 http://playground.tensorflow.org (03.10.2017).

14 The HiggsML Challenge 2014, Blogpost (https://higgsml.lal.in2p3.fr, 03.10.2017).

\section{Literatur}

Bally, Gustav 1966. Vom Spielraum der Freiheit. Die Bedeutung des Spiels bei Tier und Mensch. Stuttgart: Schwabe.

Barr, Alan J., Andy Haas und Charles W. Kalderon 2016. „That looks weird“ - Evaluating Citizen Scientists' Ability to Detect unusual Features in ATLAS Images of LHC Collisions. ATL-COM-OREACH-2016-017, arXiv:1610.02214v1.

Beck, Ulrich 2005. Power in the Global Age. Cambridge: Polity Press.

Beller, Mara 1999. Jokular Commemorations: The Copenhagen Spirit. Osiris (14): 252-273.

Bird-Davis, Nurit 2017. Before Nation: Scale-Blind Anthropology and Forager's Worlds of Relatives. Current Anthropology (58/2): 209-226.

Boisot, Max, Michael Nordberg, Saïd Yami und Bertrand Nicquevert 2011. Collisions and Collaboration. The Organization of Learning in the ATLAS Experiment at the LHC. Oxford: Oxford University Press. 
Borrelli, Arianna 2015. Was Sie schon immer über das CERN wissen wollten, aber bisher nicht zu fragen wagten - Eine philosophische und soziologische Perspektive. In: Christoph Kommer (Hg.). Großforschung in neuen Dimensionen. Berlin: Springer: 119-150.

Bourdieu, Pierre 1987. Sozialer Sinn. Kritik der theoretischen Vernunft. Frankfurt am Main: Suhrkamp.

Caillois, Roger 2001 [1958]. Man, Play and Games. Chicago: University of Illinois Press.

Carson, Cathryn 2010. Heisenberg in the Atomic Age. Science and the Public Sphere. New York: Cambridge University Press.

Cheah, Pheng und Bruce Robbins, Bruce 1998. Cosmopolitics: Thinking and Feeling beyond the Nation. Minneapolis: University of Minnesota Press.

Collier, Stephen J. und Aihwa Ong 2008. Global Assemblages, Anthropological Problems. In: Stephen J. Collier und Aihwa Ong (Hg.). Global Assemblages. Technology, Politics, and Ethics as Anthropological Problems. New York: Wiley: 3-21.

Deleuze, Gilles und Felix Guattari 1987. A Thousand Plateaus. Minneapolis: University of Minnessota Press.

Dippel, Anne und Sonia Fizek 2017. Playbouring Cyborgs@CERN. Renegotiating HumanMachine Ensembles. Vortrag auf der Konferenz der American Association of Geographers, Boston.

Dippel, Anne und Lukas Mairhofer 2017. Traces and Patterns. Pictures of Interferences and Collisions in the Physics Lab. A Dialogue Between Dr. Anne Dippel and Lukas Mairhofer. In: Bettina Bock von Wülffingen (Hg.). Traces. Generating What Was There. Berlin: De Gruyter: 75-88.

Disco, Nil, und Eda Karanakis (Hg.) 2013. Cosmopolitan Commons. Sharing Resources and Risks across Borders. Cambridge, MA: MIT Press.

Felt, Ulrike, Helga Nowotny and Klaus Taschwer 1995. Wissenschaftsforschung. Eine Einführung. Frankfurt am Main: Campus.

Findlen, Paula 1998. Between Carnival and Lent. The Scientific Revolution at the Margins of Culture. Configurations (6/2): 243-267.

Forman, Paul 1989. Social Niche and Self-Image of the American Physicist. In: Michelangelo de Maria, Mario Grilli und Fabio Sebastiani (Hg.). The Restructuring of Physical Sciences in Europe and the United States 1945-1960. Singapore: World Scientific Press: 96-104.

Galison, Peter 1997. Image and Logic. A Material Culture of Microphysics. Chicago: University of Chicago Press.

Geertz, Clifford 1973. Deep Play. Notes on the Balinese Cockfight. In: Selected Essays. New York: Basic Books: 412-454.

Geertz, Clifford 1980. Negara: The Theatre State in Nineteenth-Century Bali. Princeton, NJ: Princeton University Press.

Gillies, James und Robert Caillau 2000. How the Web Was Born. The Story of the World Wide Web. New York: Oxford University Press.

Graeber, David 2013. It is Value that Brings Universes into Being. In: $H A U$ (3/2): 219-243.

Graeber, David 2015. The Utopia of Rules. On Technology, Stupidity, and the Secret Joys of Bureaucracy. London: Melville House.

Graßhoff, Gerd und Adrian Wüthrich (Hg.) 2012. MetaATLAS. Studien zur Generierung, Validierung und Kommunikation von Wissen in einer modernen Forschungskollaboration. Bern: Bern Studies.

Hackett, Edward J. 2005. Introduction: Special Guest-edited Issue on Scientific Collaboration. Social Studies of Science (35/5): 667-671.

Hagstrom, Warren O. 1965. The Scientific Community. New York: Basic Books.

Haraway, Donna 1991. A Cyborg Manifesto: Science, Technology, and Socialist-Feminism in the Late Twentieth Century. In: Donna Haraway (Hg.). Simians, Cyborgs and Women: The Reinvention of Nature. New York: Routledge: 149-181.

Havelock, Eric A. 1963. Preface to Plato. Cambridge, MA: Belknap Press of Harvard University Press.

Houdart, Sophie 2013. The Noise of the World: The Apocalypse and the Crazy Farm Scenario. Limn, 06.2017. URL: http://limn.it/the-noise-of-the-world-the-apocalypse-andthe-crazy-farm-scenario/ (03.10.2017).

Huizinga, Johan 1992 [1938]. Homo Ludens: A Study of the Play-element in Culture. Boston: Beacon Press. 
Jasanoff, Sheila 2004. States of Knowledge. The Co-production of Science and Social Order. New York: Routledge.

Jasanoff, Sheila und Sang-Hyun Kim 2013. Sociotechnical Imaginaries and National Energy Policies. Science as Culture (22/2): 189-196.

Kasperowski, Dick und Thomas Hilman 2017. The Culture of Contribution in Citizen Science: Programs and Anti-Programs. Vortrag auf der Konferenz der Society for Social Studies of Science, Boston.

Knecht, Michi 2013. Nach Writing Culture, mit Actor-Network: Ethnographie/Praxeographie im Feld der Wissenschafts- und Technikanthropologie. In: Sabine Hess, Maria Schwertl und Hannes Moser (Hg.). Neue Perspektiven volkskundlicher/ethnologischer Methoden. Berlin: Reimer: 79-106.

Knorr-Cetina, Karin 1984. Die Fabrikation von Erkenntnis. Zur Anthropologie der Naturwissenschaft. Frankfurt am Main: Suhrkamp.

Knorr-Cetina, Karin 1999. Epistemic Cultures. How the Sciences Make Knowledge. Cambridge, MA: Harvard University Press.

Krige, John (Hg.) 1996. History of CERN. Volume III. The Years of Consolidation 1966-1980. Amsterdam: Elsevier.

Krige, John 2006. American Hegemony and the Postwar Reconstruction of Science in Europe. Cambridge, MA: MIT Press.

Krige, John 2016. Sharing Knowledge, Shaping Europe. US Technological Collaboration and Nonproliferation. Cambridge, MA: MIT Press.

Hermann, Armin, John Krige, Ulrike Mersits und Dominique Pestre 1987. History of CERN. Volume I. Launching the European Organisation for Nuclear Research. Amsterdam: North Holland.

Hermann, Armin, John Krige, Ulrike Mersits und Dominique Pestre 1990. History of CERN. Volume II. Building and Running the Laboratory 1954-1965. Amsterdam: North Holland.

Latour, Bruno und Steven Woolgar 1986. Laboratory Life. The Construction of Scientific Facts. Princeton NJ: Princeton University Press.

Lederman, Leon 2006. The God Particle. If the Universe is the Answer, what is the Question? New York: Houghton Mifflin.

Malaby, Thomas M. 2009. Anthropology and Play: The Contours of Playful Experience. New Literary History 40 (1): 205-218.

Marcuse, Herbert 1933 [1965]. Über die philosophischen Grundlagen des wirtschaftswissenschaftlichen Arbeitsbegriffs. Frankfurt am Main: Suhrkamp: 7-48.

Merz, Martina 2002. Kontrolle - Widerstand - Ermächtigung: Wie Simulationssoftware Physiker konfiguriert. In: Werner Rammert und Ingo Schulz-Schaeffer (Hg.). Können Maschinen handeln? Soziologische Beiträge zum Verhältnis von Mensch und Technik. Frankfurt am Main: Campus: 267-290.

Merz, Martina und Karin Knorr-Cetina 1994. Deconstructing in a „Thinking Science“: Theoretical Physicists at Work. Cern Theory Division (CERN-TH.7152/94).

Nickelsen, Kärin und Fabian Krämer 2016. Introduction: Cooperation and Competition in the Sciences. NTM (24/2): 119-123.

Niewöhner, Jörg, Estrid Sørensen und Stefan Beck 2012. Science and Technology Studies aus sozial- und kulturanthropologischer Perspektive. In: Jörg Niewöhner, Estrid Sørensen und Stefan Beck (Hg.). Science and Technology Studies. Eine sozialanthropologische Einführung. Bielefeld: transcript: 9-40.

Nothnagel, Dethlev 2001. The Physics way. Nationale Stile, Geschlechter und kommunikative Praxis einer internationalen Wissenschaftskultur. Frankfurt am Main: Campus.

Nowotny, Helga 2006. Cultures of Technology and the Quest for Innovation. New York: Berghahn Books.

Nowotny, Helga 2008. Insatiable Curiosity. Innovation in a Fragile Future. Cambridge, MA: MIT Press.

O’Donnell, Chris 2014. The Developer's Dilemma. The Secret World of Video Game Creators. Cambridge, MA: MIT Press.

Parkman, Anna 2016. The Imposter Phenomenon in Higher Education: Incidence and Impact. Journal of Higher Education Theory and Practice (16/1): 51-60.

Popper, Karl. 1935. Logik der Forschung. Wien: Springer. 
Pralavorio, Corinne 2016. In Practice. Are Physicists Architects of Science? CERN Homepage. URL: http://home.cern/about/updates/2016/11/practice-are-physicists-architectsscience (03.10.2017).

Roy, Arpita 2012. Science and the Large Hadron Collider: A Probe Into Instrumentation, Periodization and Classification. Dialect Anthropology (36): 291-316.

Roy, Arpita 2014. Ethnography and Theory of the Signature in Physics. Cultural Anthropo$\log y$ (29/3): 479-502.

Sahlins, Marshall 1996. The Sadness of Sweetness. The Native Anthropology of Western Cosmology. Current Anthropology (37/3): 395-428.

Schwanhäußer, Anja 2015. Herumhängen. Stadtforschung aus der Subkultur. Zeitschrift für Volkskunde (111/1): 76-93.

Shrum, Wesley 2005. Reagency of the Internet. Or, How I Became a Guest of Science. Social Studies of Science (35/5): 723-754.

Shrum, Wesley, Joel Genuth und Ivan Chompalov 2007. Structures of Scientific Collaboration Cambridge, MA: MIT University Press.

Star, Susan und James Griesemer 1989. Institutional Ecology, „Translations“ and Boundary Objects: Amateurs and Professionals in Berkeley's Museum of Vertebrate Zoology, 1907-39. Social Studies of Science (19/3): 387-420.

Steingart, Alma 2012. A Group Theory of Group Theory: Collaborative Mathematics and the „Uninvention“ of a 1000-Page Proof. Social Studies of Science (42/2): 185-213.

Stevens, Phillipps 1980. Play and Work: A False Dichotomy? In: Helen B. Schwartzmann (Hg.). Play and Culture. Westpoint NY: Leisure Press: 316-323.

Strasser, Bruno 2009. The Co-Production of Neutral Science and Neutral State in Cold War Europe: Switzerland and International Scientific Cooperation, 1951-1969. Osiris (24): 165-187.

Taylor, T.L. 2012. Raising the Stakes. E-Sports and the Professionalization of Computer Gaming. Cambridge, MA: The MIT Press.

Terranova, Tiziana 2012. Free Labor. In: Trebor Scholz (Hg.). Digital Labour. The Internet as Playground and Factory. New York, NY: Routledge: 33-57.

Traweek, Sharon 1988. Beamtimes and Lifetimes. The World of High-Energy Physics Cambridge, MA: Harvard University Press.

Turner, Victor 1999 [1969]. The Ritual Process: Structure and Anti-Structure. Chicago: Aldine.

Turner, Victor 2009. Vom Ritual zum Theater: Der Ernst des menschlichen Spiels. Frankfurt a.M.: Campus.

Vertesi, Janet 2014. Seamful Spaces: Heterogeneous Infrastructures in Interaction. Science, Technolgy E Human Values (39/2): 264-284.

Anne Dippel

Lehrstuhl für Volkskunde (Empirische Kulturwissenschaft), Institut für Kunst- und Kulturwissenschaften

Friedrich-Schiller Universität Jena

Zwätzengasse 3

07743 Jena

Deutschland

anne.dippel@uni-jena.de 